\title{
EFFICIENT AND ADAPTIVE LINEAR REGRESSION IN SEMI-SUPERVISED SETTINGS ${ }^{1}$
}

\author{
By ABHISHEK CHAKRABORTTY² AND TIANXI CAI \\ University of Pennsylvania and Harvard University
}

We consider the linear regression problem under semi-supervised settings wherein the available data typically consists of: (i) a small or moderate sized "labeled" data, and (ii) a much larger sized "unlabeled" data. Such data arises naturally from settings where the outcome, unlike the covariates, is expensive to obtain, a frequent scenario in modern studies involving large databases like electronic medical records (EMR). Supervised estimators like the ordinary least squares (OLS) estimator utilize only the labeled data. It is often of interest to investigate if and when the unlabeled data can be exploited to improve estimation of the regression parameter in the adopted linear model.

In this paper, we propose a class of "Efficient and Adaptive SemiSupervised Estimators" (EASE) to improve estimation efficiency. The EASE are two-step estimators adaptive to model mis-specification, leading to improved (optimal in some cases) efficiency under model mis-specification, and equal (optimal) efficiency under a linear model. This adaptive property, often unaddressed in the existing literature, is crucial for advocating "safe" use of the unlabeled data. The construction of EASE primarily involves a flexible "semi-nonparametric" imputation, including a smoothing step that works well even when the number of covariates is not small; and a follow up "refitting" step along with a cross-validation (CV) strategy both of which have useful practical as well as theoretical implications towards addressing two important issues: under-smoothing and over-fitting. We establish asymptotic results including consistency, asymptotic normality and the adaptive properties of EASE. We also provide influence function expansions and a "double" $\mathrm{CV}$ strategy for inference. The results are further validated through extensive simulations, followed by application to an EMR study on auto-immunity.

1. Introduction. In recent years, semi-supervised learning (SSL) has emerged as an exciting new area of research in statistics and machine learning. A detailed discussion on SSL including its practical relevance, the primary question of interest in SSL, and the existing relevant literature can be found in Chapelle, Schölkopf

Received January 2017.

${ }^{1}$ Supported in part by National Institutes of Health Grants R01 HL089778, U54 HG007963 and U01 HL121518.

${ }^{2}$ Corresponding author.

MSC2010 subject classifications. $62 \mathrm{~F} 35,62 \mathrm{~J} 05,62 \mathrm{~F} 12,62 \mathrm{G} 08$.

Key words and phrases. Semi-supervised linear regression, semiparametric inference, model misspecification, adaptive estimation, semi-nonparametric imputation. 
and Zien (2006) and Zhu (2008). A typical semi-supervised (SS) setting is characterized by two types of available data: (i) a small or moderate sized "labeled" data, $\mathcal{L}$, containing observations for both an outcome $Y$ and a set of covariates $\mathbf{X}$ of interest, and (ii) an "unlabeled" data, $\mathcal{U}$, of much larger size but having observations only for the covariates $\mathbf{X}$. By virtue of its large size, $\mathcal{U}$ essentially gives us the distribution of $\mathbf{X}$, denoted henceforth by $\mathbb{P}_{\mathbf{X}}$. Such a setting arises naturally whenever the covariates are easily available so that unlabeled data is plentiful, but the outcome is costly or difficult to obtain, thereby limiting the size of $\mathcal{L}$. This scenario is directly relevant to a variety of practical problems, especially in the modern "big data" era, with massive unlabeled datasets (often electronically recorded) becoming increasingly available and tractable. A few familiar examples include machine learning problems like text mining, web page classification, speech recognition, natural language processing, etc.

Among biomedical applications, a particularly interesting problem where SSL can be of great use is the statistical analysis of electronic medical records (EMR) data. Endowed with a wealth of de-identified clinical and phenotype data for large patient cohorts, EMR linked with bio-repositories are increasingly gaining popularity as rich resources of data for discovery research [Kohane (2011)]. Such large scale datasets obtained in a cost-effective and timely manner are of great importance in modern medical research for addressing important questions such as the biological role of genetic variants in disease susceptibility and progression [Kohane (2011)]. However, one major bottleneck impeding EMR driven research is the difficulty in obtaining validated phenotype information [Liao et al. (2010)] since they are labor intensive or expensive to obtain. Thus, gold standard labels and genomic measurements are typically available only for a small subset nested within a large cohort. In contrast, digitally recorded data on the clinical variables are often available on all subjects, highlighting the necessity and utility of developing robust SSL methods that can leverage such rich source of auxiliary information to improve phenotype definition and estimation precision.

SSL primarily distinguishes from standard supervised methods by making use of $\mathcal{U}$, an information that is ignored by the latter. The ultimate question of interest in SSL is to investigate if and when the information on $\mathbb{P}_{\mathbf{X}}$ in $\mathcal{U}$ can be exploited to improve the efficiency over a given supervised approach. In recent years, several graph based nonparametric SSL approaches have been proposed [Belkin, Niyogi and Sindhwani (2006), Zhu (2005)] for regression or classification. These approaches essentially target nonparametric SS estimation of $\mathbb{E}(Y \mid \mathbf{X})$ and, therefore, for provable improvement guarantees, must rely implicitly or explicitly on assumptions relating $\mathbb{P}_{\mathbf{X}}$ to $\mathbb{P}_{Y \mid \mathbf{X}}$ (the conditional distribution of $Y$ given $\mathbf{X}$ ), as duly noted and characterized more formally in Lafferty and Wasserman (2007). For nonparametric classification problems, the theoretical underpinnings of SSL including its scope and the consequences of using $\mathcal{U}$ have been also studied earlier by Castelli and Cover $(1995,1996)$. More parametric SS approaches, still aimed 
mostly at prediction, have also been studied for classification, including the "generative model" approach [Nigam (2001), Nigam et al. (2000)], which is based on modeling the joint distribution of $(Y, \mathbf{X})$ as an identifiable mixture of parametric models, thereby implicitly relating $\mathbb{P}_{Y \mid \mathbf{X}}$ and $\mathbb{P}_{\mathbf{X}}$. However, these approaches depend strongly on the validity of the assumed mixture model, violation of which can actually degrade their performance compared to the supervised approach [Cozman and Cohen (2001), Cozman, Cohen and Cirelo (2003)].

However, SS estimation problems, especially from a semiparametric point of view, has been somewhat less studied in SSL. Such problems are generally aimed at estimating some (finite-dimensional) parameter $\theta_{0} \equiv \theta_{0}(\mathbb{P})$, where $\mathbb{P}=\left(\mathbb{P}_{Y \mid \mathbf{X}}, \mathbb{P}_{\mathbf{X}}\right)$, and the key to the potential usefulness of $\mathcal{U}$ in improving estimation of $\theta_{0}$ lies in understanding when $\theta_{0}(\mathbb{P})$ relates to $\mathbb{P}_{\mathbf{X}}$. For simple parameters like $\theta_{0}(\mathbb{P})=\mathbb{E}(Y)$, unless $\mathbb{E}(Y \mid \mathbf{X})$ is a constant, $\theta_{0}$ clearly depends on $\mathbb{P}_{\mathbf{X}}$, and hence, improved SS estimation is possible compared to the supervised estimator $\bar{Y}_{\mathcal{L}}$, the sample mean of $Y$ based on $\mathcal{L}$. The situation is however more subtle for other choices of $\theta_{0}$, especially those where $\theta_{0}$ is the target parameter corresponding to an underlying parametric working model for $\mathbb{P}_{Y \mid \mathbf{X}}$. This includes the least squares parameter, as studied in this paper, targeted by a working linear model for $\mathbb{E}(Y \mid \mathbf{X})$. Such models are often adopted due to their appealing simplicity and interpretability.

In general, for such cases, if the adopted working model for $\mathbb{P}_{Y \mid \mathbf{X}}$ is correct and $\theta_{0}$ is not related to $\mathbb{P}_{\mathbf{X}}$, then one cannot possibly gain through SSL by using the knowledge of $\mathbb{P}_{\mathbf{X}}$ [Seeger (2002), Zhang and Oles (2000)]. On the other hand, under model mis-specification, $\theta_{0}$ may inherently depend on $\mathbb{P}_{\mathbf{X}}$, and thus imply the potential utility of $\mathcal{U}$ in improving the estimation. However, inappropriate use of $\mathcal{U}$ may lead to degradation of the estimation precision. This therefore signifies the need for robust and efficient SS estimators that are adaptive to model mis-specification, so that they are as efficient as the supervised estimator under the correct model and more efficient under model mis-specification. To the best of our knowledge, work done along these lines is relatively scarce in the SSL literature, one notable exception being the recent work of Kawakita and Kanamori (2013), where they use a very different approach based on density ratio estimation, building on the more restrictive approach of Sokolovska, Cappé and Yvon (2008). However, as we observe in our simulation studies, the extent of the efficiency gain actually achieved by these approaches can be quite incremental, at least in finite samples. Further, the seemingly unclear choice of the ideal (nuisance) model to be used for density ratio estimation can also have a significant impact on the performance, both finite sample and asymptotic, of these estimators.

We propose here a class of Efficient and Adaptive Semi-Supervised Estimators (EASE) in the context of linear regression problems. We essentially adopt a semiparametric perspective wherein the adopted linear "working" model can be potentially mis-specified, and the goal is to obtain efficient and adaptive SS estimators of the regression parameter through robust usage of $\mathcal{U}$. The EASE are 
two-step estimators with a simple and scalable construction based on a first step of "semi-nonparametric" (SNP) imputation, which includes a smoothing step and a follow-up "refitting" step. In the second step, we regress the imputed outcomes against the covariates using the unlabeled data to obtain our SNP imputation based SS estimator, and then further combine it optimally with the supervised estimator to obtain the final EASE. Dimension reduction methods are also employed in the smoothing step to accommodate higher dimensional $\mathbf{X}$, if necessary. Further, we extensively adopt cross-validation (CV) techniques in the imputation, leading to some useful theoretical properties (apart from practical benefits) typically not observed for smoothing based two-step estimators. We demonstrate that EASE is guaranteed to be efficient and adaptive in the sense discussed above, and also achieves semiparametric optimality whenever the SNP imputation is "sufficient" or the linear model holds. We also provide data adaptive methods to optimally select the directions for smoothing when dimension reduction is desired, and tools for inference with EASE.

The rest of this paper is organized as follows. In Section 2, we formulate the SS linear regression problem. In Section 3, we construct a family of SS estimators based on SNP imputation and establish all their properties, and further propose the EASE as a refinement of these estimators. For all our proposed estimators, we also address their associated inference procedures based on "double" CV methods. In Section 4, we discuss a kernel smoothing based implementation of the SNP imputation and establish all its properties. In Section 5, we discuss SS dimension reduction techniques, useful for implementing the SNP imputation. Simulation results and an application to an EMR study are shown in Section 6, followed by concluding discussions in Section 7. Proofs of all theoretical results and associated technical materials, and further numerical results and discussions are distributed in the Appendix and the Supplementary Material [Chakrabortty and Cai (2018)].

\section{Problem set-up.}

Data representation. Let $Y \in \mathbb{R}$ denote the outcome random variable and $\mathbf{X} \in \mathbb{R}^{p}$ denote the covariate vector, where $p$ is fixed, and let $\mathbf{Z}=\left(Y, \mathbf{X}^{\prime}\right)^{\prime}$. Then the entire data available for analysis can be represented as $\mathbb{S}=(\mathcal{L} \cup \mathcal{U})$, where $\mathcal{L}=\left\{\mathbf{Z}_{i} \equiv\left(Y_{i}, \mathbf{X}_{i}^{\prime}\right)^{\prime}: i=1, \ldots, n\right\}$ consists of $n$ independent and identically distributed (i.i.d.) observations from the joint distribution $\mathbb{P}_{\mathbf{Z}}$ of $\mathbf{Z}, \mathcal{U}=\left\{\mathbf{X}_{i}\right.$ : $i=n+1, \ldots, n+N\}$ consists of $N$ i.i.d. observations from $\mathbb{P}_{\mathbf{X}}$, and $\mathcal{L} \Perp \mathcal{U}$. Throughout, for notational convenience, we use the subscript " $j$ " to denote the unlabeled observations, and re-index without loss of generality (w.l.o.g.) the $N$ observations in $\mathcal{U}$ as: $\mathcal{U}=\left\{\mathbf{X}_{j}: j=n+1, \ldots, n+N\right\}$.

Assumption 2.1 (Basic assumptions). (a) We assume that $\mathbf{Z}$ has finite 2 nd moments and $\boldsymbol{\Sigma} \equiv \operatorname{Var}(\mathbf{X})$ is positive definite, denoted as $\boldsymbol{\Sigma} \succ 0$. We also assume, for simplicity, that $\mathbf{X}$ has a compact support $\mathcal{X} \subseteq \mathbb{R}^{p}$. 
(b) We assume $N \gg n$, that is, $n / N \rightarrow 0$ as $n, N \rightarrow \infty$, and $\mathcal{L}$ and $\mathcal{U}$ arise from the same underlying distribution, that is, $\mathbf{Z} \sim \mathbb{P}_{\mathbf{Z}}$ for all subjects in $\mathcal{S}$.

Notation. Let $\boldsymbol{\Gamma}=\mathbb{E}\left(\overrightarrow{\mathbf{X}} \overrightarrow{\mathbf{X}}^{\prime}\right) \succ 0$, where $\forall \mathbf{v} \in \mathbb{R}^{p}, \overrightarrow{\mathbf{v}}=\left(1, \mathbf{v}^{\prime}\right)^{\prime} \in \mathbb{R}^{(p+1)}$. Let $\mathcal{L}_{2}\left(\mathbb{P}_{\mathbf{X}}\right)$ denote the space of all $\mathbb{R}$-valued measurable functions of $\mathbf{X}$ having finite $L_{2}$ norm with respect to (w.r.t.) $\mathbb{P}_{\mathbf{X}}$, and for any $g(\cdot) \in \mathcal{L}_{2}\left(\mathbb{P}_{\mathbf{X}}\right)$, let $\boldsymbol{\Sigma}(g) \succ 0$ denote the $(p+1) \times(p+1)$ matrix $\boldsymbol{\Gamma}^{-1} \mathbb{E}\left[\overrightarrow{\mathbf{X}} \overrightarrow{\mathbf{X}}^{\prime}\{Y-g(\mathbf{X})\}^{2}\right] \boldsymbol{\Gamma}^{-1}$. Lastly, let $\|\cdot\|$ denote the $L_{2}$ vector norm, and for any integer $a \geq 1$, let $I_{a}$ denote the identity matrix of order $a$, and $\mathcal{N}_{a}[\boldsymbol{\mu}, \boldsymbol{\Omega}]$ denote the $a$-variate Gaussian distribution with mean $\boldsymbol{\mu} \in \mathbb{R}^{a}$ and covariance matrix $\boldsymbol{\Omega}_{a \times a} \succ 0$.

REMARK 2.1. Assumption 2.1(b) enlists some fundamental characteristics of SS settings. Indeed, the condition of $\mathcal{L}$ and $\mathcal{U}$ being equally distributed has usually been an integral part of the definition of SS settings [Chapelle, Schölkopf and Zien (2006), Kawakita and Kanamori (2013)]. Interpreted in missing data terminology, it entails that $Y$ in $\mathcal{U}$ are "missing completely at random" (MCAR), with the missingness/labeling being typically by design. Interestingly, the crucial assumption of MCAR, although commonly required, has often stayed implicit in the SSL literature [Lafferty and Wasserman (2007)]. It is important to note that while the SS set-up can be viewed as a missing data problem, it is quite different from standard ones, since with $n / N \rightarrow 0$, that is, $|\mathcal{U}| \gg|\mathcal{L}|$, the proportion of $Y$ observed in $\mathcal{S}$ tends to 0 in SSL. Hence, the "positivity assumption" typical in missing data theory, requiring this proportion to be bounded away from 0 , is violated here. It is also worth noting that owing to such violations, the analysis of SS settings under more general missingness mechanisms such as "missing at random" (MAR) is considerably more complicated and to our knowledge, the literature for SS estimation problems under such settings is virtually nonexistent. Furthermore, for such problems, the traditional goal in SSL, that of improving upon a "supervised" estimator, can become unclear without MCAR, unless an appropriately weighted version of the supervised estimator is considered. Given these subtleties and the traditional assumptions (often implicit) in SSL, the MCAR condition is assumed for most of this paper, although a brief discussion on possible extensions of our proposed SS estimators to MAR settings is provided in the Supplementary Material [Chakrabortty and Cai (2018)].

2.1. The target parameter and its supervised estimator. We consider the linear regression working model given by

$$
Y=\overrightarrow{\mathbf{X}}^{\prime} \boldsymbol{\theta}+\varepsilon, \quad \text { with } \mathbb{E}(\varepsilon \mid \mathbf{X})=0,
$$

where $\boldsymbol{\theta} \in \mathbb{R}^{(p+1)}$ is an unknown regression parameter. Accounting for the potential mis-specification of the working model (2.1), we define the target parameter of interest as a model-free parameter, as follows: 
DEFINITION 2.1. The target parameter $\boldsymbol{\theta}_{0}$ for linear regression may be defined as the solution to the normal equations: $\mathbb{E}\left\{\overrightarrow{\mathbf{X}}\left(Y-\overrightarrow{\mathbf{X}}^{\prime} \boldsymbol{\theta}\right)\right\}=\mathbf{0}$ in $\boldsymbol{\theta} \in \mathbb{R}^{(p+1)}$, or equivalently, $\boldsymbol{\theta}_{0}=\operatorname{argmin}_{\boldsymbol{\theta} \in \mathbb{R}^{(p+1)}} \mathbb{E}\left(Y-\overrightarrow{\mathbf{X}}^{\prime} \boldsymbol{\theta}\right)^{2}$.

Existence and uniqueness of $\boldsymbol{\theta}_{0}$ in Definition 2.1 is clear. Further, $\overrightarrow{\mathbf{X}}^{\prime} \boldsymbol{\theta}_{0}$ is the $L_{2}$ projection of $\mathbb{E}(Y \mid \mathbf{X}) \in \mathcal{L}_{2}\left(\mathbb{P}_{\mathbf{X}}\right)$ onto the subspace of all linear functions of $\mathbf{X}$, and hence, is the best linear predictor of $Y$ given $\mathbf{X}$. The linear model (2.1) is correct (else, mis-specified) if and only if $\mathbb{E}(Y \mid \mathbf{X})$ lies in this space [in which case, $\mathbb{E}(Y \mid \mathbf{X})=\overrightarrow{\mathbf{X}}^{\prime} \boldsymbol{\theta}_{0}$ ]. When the model is correct, $\boldsymbol{\theta}_{0}$ depends only on $\mathbb{P}_{Y \mid \mathbf{X}}$, not on $\mathbb{P}_{\mathbf{X}}$. Hence, improved estimation of $\boldsymbol{\theta}_{0}$ through SSL is impossible in this case unless further assumptions relating $\boldsymbol{\theta}_{0}$ to $\mathbb{P}_{\mathbf{X}}$ are made. On the other hand, under model mis-specification, the normal equations defining $\boldsymbol{\theta}_{0}$ inherently depend on $\mathbb{P}_{\mathbf{X}}$, thereby implying the potential utility of SSL in improving the estimation of $\boldsymbol{\theta}_{0}$ in this case.

The usual supervised estimator of $\boldsymbol{\theta}_{0}$ is the OLS estimator $\widehat{\boldsymbol{\theta}}$, the solution in $\boldsymbol{\theta}$ to the equation: $n^{-1} \sum_{i=1}^{n} \overrightarrow{\mathbf{X}}_{i}\left(Y_{i}-\overrightarrow{\mathbf{X}}_{i}^{\prime} \boldsymbol{\theta}\right)=\mathbf{0}$, the normal equations based on $\mathcal{L}$. Under Assumption 2.1(a), it is well known that as $n \rightarrow \infty$,

$$
n^{\frac{1}{2}}\left(\widehat{\boldsymbol{\theta}}-\boldsymbol{\theta}_{0}\right)=n^{-\frac{1}{2}} \sum_{i=1}^{n} \boldsymbol{\psi}_{0}\left(\mathbf{Z}_{i}\right)+O_{p}\left(n^{-\frac{1}{2}}\right) \stackrel{d}{\rightarrow} \mathcal{N}_{(p+1)}\left[\mathbf{0}, \boldsymbol{\Sigma}\left(g_{\boldsymbol{\theta}_{0}}\right)\right],
$$

where $\boldsymbol{\psi}_{0}(\mathbf{Z})=\boldsymbol{\Gamma}^{-1}\left\{\overrightarrow{\mathbf{X}}\left(Y-\overrightarrow{\mathbf{X}}^{\prime} \boldsymbol{\theta}_{0}\right)\right\}$ and $g_{\boldsymbol{\theta}}(\mathbf{X})=\overrightarrow{\mathbf{X}}^{\prime} \boldsymbol{\theta} \forall \boldsymbol{\theta} \in \mathbb{R}^{(p+1)}$.

Our primary goal is to obtain efficient SS estimators of $\boldsymbol{\theta}_{0}$ using the entire data $\mathcal{S}$ and compare their efficiencies to that of $\widehat{\boldsymbol{\theta}}$. It is worth noting that the estimation efficiency of $\boldsymbol{\theta}_{0}$ also relates to the predictive performance of the fitted linear model since its out-of-sample prediction error is directly related to the mean squared error (w.r.t. the $\Sigma$ metric) of the parameter estimate.

3. A family of imputation based semi-supervised estimators. If $Y$ in $\mathcal{U}$ were actually observed, then one would simply fit the working model to the entire data in $\mathcal{S}$ for estimating $\boldsymbol{\theta}_{0}$. Our general approach is precisely motivated by this intuition. We first attempt to impute the missing $Y$ in $\mathcal{U}$ based on suitable training of $\mathcal{L}$ in step (I). Then in step (II), we fit the linear model (2.1) to $\mathcal{U}$ with the imputed outcomes. Clearly, the imputation is critical. Inaccurate imputation would lead to biased estimate of $\boldsymbol{\theta}_{0}$, while inadequate imputation would result in loss of efficiency. We next consider SS estimators constructed under two imputation strategies for step (I) including a fully nonparametric imputation based on kernel smoothing (KS), and a semi-nonparametric (SNP) imputation that involves a smoothing step and a follow up "refitting" step. Although the construction of the final EASE is based on the SNP imputation strategy, it is helpful to begin with a discussion of the first strategy in order to appropriately motivate and elucidate the discussion on EASE and the SNP imputation strategy. 
3.1. A simple SS estimator via fully nonparametric imputation. We present here an estimator based on a fully nonparametric imputation involving KS when $p$ is small. For simplicity, we shall assume here that $\mathbf{X}$ is continuous with a density $f(\cdot)$. Let $m(\mathbf{x})=\mathbb{E}(Y \mid \mathbf{X}=\mathbf{x})$ and $l(\mathbf{x})=m(\mathbf{x}) f(\mathbf{x})$. Consider the local constant KS estimator of $m(\mathbf{x})$ :

$$
\widehat{m}(\mathbf{x})=\frac{\frac{1}{n h^{p}} \sum_{i=1}^{n}\left\{K_{h}\left(\mathbf{X}_{i}, \mathbf{x}\right)\right\} Y_{i}}{\frac{1}{n h^{p}} \sum_{i=1}^{n} K_{h}\left(\mathbf{X}_{i}, \mathbf{x}\right)}=\frac{\widehat{l}(\mathbf{x})}{\widehat{f}(\mathbf{x})},
$$

where $K_{h}(\mathbf{u}, \mathbf{v})=K\{(\mathbf{u}-\mathbf{v}) / h\}$ with $K: \mathbb{R}^{p} \rightarrow \mathbb{R}$ being some suitable kernel function and $h=h(n)>0$ being the bandwidth. With $\widehat{m}(\cdot)$ as defined in (3.1), we now fit (2.1) to the imputed unlabeled data: $\left[\left\{\widehat{m}\left(\mathbf{X}_{j}\right), \mathbf{X}_{j}^{\prime}\right\}^{\prime}: j=n+1, \ldots, n+N\right]$ and obtain a SS estimator $\widehat{\boldsymbol{\theta}}_{n p}$ of $\boldsymbol{\theta}_{0}$ as the solution in $\boldsymbol{\theta}$ to

$$
\frac{1}{N} \sum_{j=n+1}^{n+N} \overrightarrow{\mathbf{X}}_{j}\left\{\widehat{m}\left(\mathbf{X}_{j}\right)-\overrightarrow{\mathbf{X}}_{j}^{\prime} \boldsymbol{\theta}\right\}=\mathbf{0} .
$$

Here and throughout in our constructions of SS estimators, $\mathcal{L}$ with either the true or the imputed $Y$ is not included in the final fitting step mostly due to technical convenience in the asymptotic analysis of our estimators, and also due to the fact that the contribution of $\mathcal{L}$, included in any form, in the final fitting step is asymptotically negligible since $n / N \rightarrow 0$.

In order to study the properties of $\widehat{\boldsymbol{\theta}}_{n p}$, we require uniform (in $L_{\infty}$ norm) convergence of $\widehat{m}(\cdot)$ to $m(\cdot)$, a problem that has been extensively studied in the nonparametric statistics literature [Andrews (1995), Hansen (2008), Masry (1996), Newey (1994)] under fairly general settings and assumptions. In particular, we would assume the following regularity conditions to hold.

ASSUMPTION 3.1. (i) $K(\cdot)$ is a symmetric $q$ th order kernel for some integer $q \geq 2$. (ii) $K(\cdot)$ is bounded, Lipschitz continuous and has a bounded support $\mathcal{K} \subseteq$ $\mathbb{R}^{p}$. (iii) $\mathbb{E}\left(|Y|^{s}\right)<\infty$ for some $s>2$. $\mathbb{E}\left(|Y|^{s} \mid \mathbf{X}=\mathbf{x}\right) f(\mathbf{x})$ and $f(\mathbf{x})$ are bounded on $\mathcal{X}$. (iv) $f(\mathbf{x})$ is bounded away from 0 on $\mathcal{X}$. (v) $m(\cdot)$ and $f(\cdot)$ are $q$ times continuously differentiable with bounded $q$ th derivatives on some open set $\mathcal{X}_{0} \supseteq$ $\mathcal{X}$. (vi) For any $\delta>0$, let $A_{\delta} \subseteq \mathbb{R}^{p}$ denote the set $\{(\mathbf{x}-\mathbf{X}) / \delta: \mathbf{x} \in \mathcal{X}\}$. Then, for small enough $\delta, A_{\delta} \supseteq \mathcal{K}$ almost surely (a.s.).

Conditions (i) $-(\mathrm{v})$ are fairly standard in the literature. In (v), the set $\mathcal{X}_{0}$ is needed mostly to make the notion of differentiability well defined, with both $m(\cdot)$ and $f(\cdot)$ understood to have been analytically extended over $\left(\mathcal{X}_{0} \backslash \mathcal{X}\right)$. Condition (vi) implicitly controls the tail behaviour of $\mathbf{X}$, requiring that perturbations of $\mathbf{X}$ in the form of $(\mathbf{X}+\delta \boldsymbol{\phi})$ with $\boldsymbol{\phi} \in \mathcal{K}$ (bounded) and $\delta$ small enough, belong to $\mathcal{X}$ a.s. $\left[\mathbb{P}_{\mathbf{X}}\right]$. We now present our result on $\widehat{\boldsymbol{\theta}}_{n p}$. 
TheOREM 3.1. Suppose $n^{\frac{1}{2}} h^{q} \rightarrow 0$ and $(\log n) /\left(n^{\frac{1}{2}} h^{p}\right) \rightarrow 0$ as $n \rightarrow \infty$, and let $r_{n}=n^{\frac{1}{2}} h^{q}+(\log n) /\left(n^{\frac{1}{2}} h^{p}\right)+(n / N)^{\frac{1}{2}}$. Then, under Assumption 3.1,

$$
n^{\frac{1}{2}}\left(\widehat{\boldsymbol{\theta}}_{n p}-\boldsymbol{\theta}_{0}\right)=n^{-\frac{1}{2}} \sum_{i=1}^{n} \boldsymbol{\psi}_{\mathrm{eff}}\left(\mathbf{Z}_{i}\right)+O_{p}\left(r_{n}\right) \stackrel{d}{\rightarrow} \mathcal{N}_{(p+1)}[\mathbf{0}, \boldsymbol{\Sigma}(m)],
$$

where $\boldsymbol{\psi}_{\mathrm{eff}}(\mathbf{Z})=\boldsymbol{\Gamma}^{-1}[\overrightarrow{\mathbf{X}}\{Y-m(\mathbf{X})\}]$.

REMARK 3.1. Theorem 3.1 establishes the efficient and adaptive nature of $\widehat{\boldsymbol{\theta}}_{n p}$. The asymptotic variance $\boldsymbol{\Sigma}(m)$ of $\widehat{\boldsymbol{\theta}}_{n p}$ satisfies $\boldsymbol{\Sigma}(g)-\boldsymbol{\Sigma}(m) \succeq 0 \forall g(\cdot) \in$ $\mathcal{L}_{2}\left(\mathbb{P}_{\mathbf{X}}\right)$ and the inequality is strict unless $g(\cdot)=m(\cdot)$ a.s. $\left[\mathbb{P}_{\mathbf{X}}\right]$. Hence, $\widehat{\boldsymbol{\theta}}_{n p}$ is asymptotically optimal among the class of all regular and asymptotically linear (RAL) estimators of $\boldsymbol{\theta}_{0}$ with influence function (IF) of the form $\boldsymbol{\Gamma}^{-1}[\overrightarrow{\mathbf{X}}\{Y-$ $g(\mathbf{X})\}]$ with $g(\cdot) \in \mathcal{L}_{2}\left(\mathbb{P}_{\mathbf{X}}\right)$. In particular, $\widehat{\boldsymbol{\theta}}_{n p}$ is more efficient than $\widehat{\boldsymbol{\theta}}$ whenever (2.1) is mis-specified, and equally efficient when (2.1) is correct, that is, $m(\cdot)=g_{\boldsymbol{\theta}_{0}}(\cdot)$. Further, it can also be shown that $\boldsymbol{\psi}_{\text {eff }}(\mathbf{Z})$ is the "efficient" IF for estimating $\boldsymbol{\theta}_{0}$ under the semiparametric model $\mathcal{M}_{\mathbf{X}} \equiv\left\{\left(\mathbb{P}_{Y \mid \mathbf{X}}, \mathbb{P}_{\mathbf{X}}\right)\right.$ : $\mathbb{P}_{\mathbf{X}}$ is known, $\mathbb{P}_{Y \mid \mathbf{X}}$ is unrestricted up to Assumption 2.1(a)\}. Thus, $\widehat{\boldsymbol{\theta}}_{n p}$ also globally achieves the semiparametric efficiency bound under $\mathcal{M}_{\mathbf{X}}$. Lastly, note that at any parametric sub-model in $\mathcal{M}_{\mathbf{X}}$ that corresponds to (2.1) being correct, $\widehat{\boldsymbol{\theta}}$ also achieves optimality, thus showing that under $\mathcal{M}_{\mathbf{X}}$, it is not possible to improve upon $\widehat{\boldsymbol{\theta}}$ if the linear model is correct.

REMARK 3.2. The asymptotic results in Theorem 3.1 require a kernel of order $q>p$ and $h$ smaller in order than the "optimal" bandwidth order $h_{\text {opt }}=$ $O\left(n^{-1 /(2 q+p)}\right)$. This under-smoothing requirement, often encountered in two-step estimators involving a first-step smoothing [Newey, Hsieh and Robins (1998)], generally results in sub-optimal performance of $\widehat{m}(\cdot)$. The optimal under-smoothed bandwidth order for Theorem 3.1 is given by $O\left(n^{-1 /(q+p)}\right)$.

3.2. SS estimators based on semi-nonparametric (SNP) imputation. The simple and intuitive imputation strategy in Section 3.1 based on a fully nonparametric $p$-dimensional KS is however often undesirable in practice owing to the curse of dimensionality. In order to accommodate larger $p$, we now propose a more flexible SNP imputation method involving a dimension reduction, if needed, followed by a nonparametric calibration. An additional "refitting" step is proposed to reduce the impact of bias from nonparametric estimation and possibly inadequate imputation due to dimension reduction. We also introduce some flexibility in terms of the smoothing methods, apart from KS, that can be used for the nonparametric calibration.

Let $r \leq p$ be a fixed positive integer and let $\mathbf{P}_{r}=\left[\mathbf{p}_{1}, \ldots, \mathbf{p}_{r}\right]_{p \times r}$ be any rank $r$ transformation matrix. Let $\mathbf{X}_{\mathbf{P}_{r}}=\mathbf{P}_{r}^{\prime} \mathbf{X}$. Given $\left(r, \mathbf{P}_{r}\right)$, we may now consider approximating the regression function $\mathbb{E}(Y \mid \mathbf{X})$ by smoothing $Y$ over the $r$ dimensional $\mathbf{X}_{\mathbf{P}_{r}}$ instead of the original $\mathbf{X} \in \mathbb{R}^{p}$. In general, $\mathbf{P}_{r}$ can be user-defined and 
data dependent. A few reasonable choices of $\mathbf{P}_{r}$ are discussed in Section 5. If $\mathbf{P}_{r}$ depends only on the distribution of $\mathbf{X}$, it may be assumed to be known given the SS setting considered. If $\mathbf{P}_{r}$ also depends on the distribution of $Y$, then it needs to be estimated from $\mathcal{L}$ and the smoothing needs to be performed using the estimated $\mathbf{P}_{r}$.

For approximating $\mathbb{E}(Y \mid \mathbf{X})$, we may consider any reasonable smoothing technique $\mathcal{T}$. Some examples of $\mathcal{T}$ include $\mathrm{KS}$, kernel machine regression and smoothing splines. Let $m\left(\mathbf{x} ; \mathbf{P}_{r}\right)$ denote the "target function" for smoothing $Y$ over $\mathbf{X}_{\mathbf{P}_{r}}$ using $\mathcal{T}$. For notational simplicity, the dependence of $m\left(\mathbf{x} ; \mathbf{P}_{r}\right)$ and other quantities on $\mathcal{T}$ is suppressed throughout. For $\mathcal{T}:=\mathrm{KS}$, the appropriate target function is given by: $m\left(\mathbf{x} ; \mathbf{P}_{r}\right)=m_{\mathbf{P}_{r}}\left(\mathbf{P}_{r}^{\prime} \mathbf{x}\right)$, where $m_{\mathbf{P}_{r}}(\mathbf{z}) \equiv \mathbb{E}\left(Y \mid \mathbf{X}_{\mathbf{P}_{r}}=\mathbf{z}\right)$. For basis function expansion based methods, $m\left(\mathbf{x} ; \mathbf{P}_{r}\right)$ will typically correspond to the $L_{2}$ projection of $m(\mathbf{x}) \equiv \mathbb{E}(Y \mid \mathbf{X}=\mathbf{x}) \in \mathcal{L}_{2}\left(\mathbb{P}_{\mathbf{X}}\right)$ onto the functional space spanned by the basis functions associated with $\mathcal{T}$. The results in this section apply to any choice of $\mathcal{T}$ that satisfies the required conditions. In Section 4, we provide more specific results for the implementation of our methods using $\mathcal{T}:=\mathrm{KS}$.

Note that we do not assume $m\left(\mathbf{x} ; \mathbf{P}_{r}\right)=m(\mathbf{x})$ anywhere, and hence the name "semi-nonparametric". Clearly, with $\mathbf{P}_{r}=I_{p}$ and $\mathcal{T}:=\mathrm{KS}$, it reduces to a fully nonparametric approach. We next describe the two sub-steps involved in step (I) of the SNP imputation: (Ia) smoothing, and (Ib) refitting.

(Ia) smoothing step. With $\mathbf{P}_{r}$ and $m\left(\mathbf{x} ; \mathbf{P}_{r}\right)$ as defined above, let $\widehat{\mathbf{P}}_{r}$ and $\widehat{m}\left(\mathbf{x} ; \widehat{\mathbf{P}}_{r}\right)$, respectively, denote their estimators based on $\mathcal{L}$. In order to address potential overfitting issues in the subsequent steps, we further consider generalized versions of these estimators based on $\mathbb{K}$-fold $\mathrm{CV}$ for a given fixed integer $\mathbb{K} \geq 1$. For any $\mathbb{K} \geq 2$, let $\left\{\mathcal{L}_{k}\right\}_{k=1}^{\mathbb{K}}$ denote a random partition of $\mathcal{L}$ into $\mathbb{K}$ disjoint subsets of equal sizes, $n_{\mathbb{K}}=n / \mathbb{K}$, with index sets $\left\{\mathcal{I}_{k}\right\}_{k=1}^{\mathbb{K}}$. Let $\mathcal{L}_{k}^{-}$denote the set excluding $\mathcal{L}_{k}$ with size $n_{\mathbb{K}}^{-}=n-n_{\mathbb{K}}$ and respective index set $\mathcal{I}_{k}^{-}$. Let $\widehat{\mathbf{P}}_{r, k}$ and $\widehat{m}_{k}\left(\mathbf{x} ; \widehat{\mathbf{P}}_{r, k}\right)$ denote the corresponding estimators based on $\mathcal{L}_{k}^{-}$. Further, for notational consistency, we define for $\mathbb{K}=1, \mathcal{L}_{k}=\mathcal{L}_{k}^{-}=\mathcal{L} ; \mathcal{I}_{k}=\mathcal{I}_{k}^{-}=\{1, \ldots, n\} ; n_{\mathbb{K}}=n_{\mathbb{K}}^{-}=n ; \widehat{\mathbf{P}}_{r, k}=\widehat{\mathbf{P}}_{r}$ and $\widehat{m}_{k}\left(\mathbf{x} ; \widehat{\mathbf{P}}_{r, k}\right)=\widehat{m}\left(\mathbf{x} ; \widehat{\mathbf{P}}_{r}\right)$.

(Ib) refitting step. In this step, we fit the linear model to $\mathcal{L}$ using $\mathbf{X}$ as predictors and the estimated $m\left(\mathbf{X} ; \mathbf{P}_{r}\right)$ as an offset. To motivate this, we recall that the fully nonparametric imputation given in Section 3.1 consistently estimates $\mathbb{E}(Y \mid \mathbf{X})$, the $L_{2}$ projection onto a space that always contains the working model space, that is, the linear span of $\overrightarrow{\mathbf{X}}$. This need not be true for the SNP imputation, since we do not assume $m\left(\mathbf{X} ; \mathbf{P}_{r}\right)=m(\mathbf{X})$ necessarily. The refitting step essentially "adjusts" for this so that the final imputation, combining the predictions from these two steps, targets a space that contains the working model space. In particular, for $\mathcal{T}:=\mathrm{KS}$ with $r<p$, this step is critical to remove potential bias due to inadequate imputation.

Interestingly, it turns out that the refitting step should always be performed, even when $m\left(\mathbf{X} ; \mathbf{P}_{r}\right)=m(\mathbf{X})$. It plays a crucial role in reducing the bias of the resulting $\mathrm{SS}$ estimator due to the inherent bias from nonparametric curve estimation. In 
particular, for $\mathcal{T}:=\mathrm{KS}$ with any $r \leq p$, it ensures that a bandwidth of the optimal order can be used, thereby eliminating the under-smoothing issue as encountered in Section 3.1. The target parameter for the refitting step is simply the regression coefficient obtained from regressing the residual $Y-m\left(\mathbf{X} ; \mathbf{P}_{r}\right)$ on $\mathbf{X}$ and may be defined as: $\boldsymbol{\eta}_{\mathbf{P}_{r}}$, the solution in $\boldsymbol{\eta} \in \mathbb{R}^{(p+1)}$ to the equation: $\mathbb{E}\left[\overrightarrow{\mathbf{X}}\left\{Y-m\left(\mathbf{X} ; \mathbf{P}_{r}\right)-\right.\right.$ $\left.\left.\overrightarrow{\mathbf{X}}^{\prime} \boldsymbol{\eta}\right\}\right]=\mathbf{0}$. For any $\mathbb{K} \geq 1$, we estimate $\boldsymbol{\eta}_{\mathbf{P}_{r}}$ as $\widehat{\boldsymbol{\eta}}_{\left(\mathbf{P}_{r}, \mathbb{K}\right)}$, the solution in $\boldsymbol{\eta}$ to the equation:

$$
n^{-1} \sum_{k=1}^{\mathbb{K}} \sum_{i \in \mathcal{I}_{k}} \overrightarrow{\mathbf{X}}_{i}\left\{Y_{i}-\widehat{m}_{k}\left(\mathbf{X}_{i} ; \widehat{\mathbf{P}}_{r, k}\right)-\overrightarrow{\mathbf{X}}_{i}^{\prime} \boldsymbol{\eta}\right\}=\mathbf{0}
$$

For $\mathbf{X}_{i} \in \mathcal{L}_{k}$, the estimate of $m\left(\mathbf{X}_{i} ; \mathbf{P}_{r}\right)$ to be used as an offset is obtained from $\widehat{m}_{k}\left(\cdot ; \widehat{\mathbf{P}}_{r, k}\right)$ that is based on data in $\mathcal{L}_{k}^{-}$. For $\mathbb{K} \geq 2$, with $\mathcal{L}_{k}^{-} \Perp \mathcal{L}_{k}$, the residuals are thus estimated in a cross-validated manner. For $\mathbb{K}=1$, however, $\widehat{m}\left(\cdot ; \widehat{\mathbf{P}}_{r}\right)$ is estimated using the entire $\mathcal{L}$ which can lead to considerable underestimation of the true residuals owing to over-fitting and consequently, substantial finite sample bias in the resulting SS estimator of $\boldsymbol{\theta}_{0}$. This bias can be effectively reduced by using the $\mathrm{CV}$ approach with $\mathbb{K} \geq 2$. We next estimate the target function for the SNP imputation given by

$$
\begin{aligned}
\mu\left(\mathbf{x} ; \mathbf{P}_{r}\right) & =m\left(\mathbf{x} ; \mathbf{P}_{r}\right)+\overrightarrow{\mathbf{x}}^{\prime} \boldsymbol{\eta}_{\mathbf{P}_{r}} \quad \text { as: } \\
\widehat{\mu}\left(\mathbf{x} ; \widehat{\mathcal{P}}_{r, \mathbb{K}}\right) & =\mathbb{K}^{-1} \sum_{k=1}^{\mathbb{K}} \widehat{m}_{k}\left(\mathbf{x} ; \widehat{\mathbf{P}}_{r, k}\right)+\overrightarrow{\mathbf{x}}^{\prime} \widehat{\boldsymbol{\eta}}_{\left(\mathbf{P}_{r}, \mathbb{K}\right)},
\end{aligned}
$$

where $\widehat{\mathcal{P}}_{r, \mathbb{K}}=\left\{\widehat{\mathbf{P}}_{r, k}\right\}_{k=1}^{\mathbb{K}}$. For notational simplicity, we suppress throughout the inherent dependence of $\widehat{\mu}(\cdot ; \cdot)$ itself on $\mathbb{K}$ and $\left\{\mathcal{L}_{k}^{-}\right\}_{k=1}^{\mathbb{K}}$. Note that similar to $m\left(\mathbf{X} ; \mathbf{P}_{r}\right)$, we also do not assume $\mu\left(\mathbf{X} ; \mathbf{P}_{r}\right)=m(\mathbf{X})$. Apart from the geometric motivation for the refitting step and its technical role in bias reduction, it also generally ensures the condition: $\mathbb{E}\left[\overrightarrow{\mathbf{X}}\left\{Y-\mu\left(\mathbf{X} ; \mathbf{P}_{r}\right)\right\}\right]=\mathbf{0}$, regardless of the true underlying $m(\mathbf{X})$. This condition is a key requirement for the asymptotic expansions, in Theorem 3.2, of our resulting SS estimators. Using $\widehat{\mu}\left(\cdot ; \widehat{\mathcal{P}}_{r, \mathbb{K}}\right)$, we now construct our final SS estimator as follows.

SS estimator from SNP imputation. In step (II), we fit the linear model to the SNP imputed unlabeled data: $\left[\left\{\widehat{\mu}\left(\mathbf{X}_{j} ; \widehat{\mathcal{P}}_{r, \mathbb{K}}\right), \mathbf{X}_{j}^{\prime}\right\}^{\prime}: j=n+1, \ldots, n+N\right]$ and obtain a $\mathrm{SS}$ estimator $\widehat{\boldsymbol{\theta}}_{\left(\mathbf{P}_{r}, \mathbb{K}\right)}$ of $\boldsymbol{\theta}_{0}$ given by

(3.7) $\widehat{\boldsymbol{\theta}}_{\left(\mathbf{P}_{r}, \mathbb{K}\right)}$ is the solution in $\boldsymbol{\theta}$ to

$$
\frac{1}{N} \sum_{j=n+1}^{n+N} \overrightarrow{\mathbf{X}}_{j}\left\{\widehat{\mu}\left(\mathbf{X}_{j} ; \widehat{\mathcal{P}}_{r, \mathbb{K}}\right)-\overrightarrow{\mathbf{X}}_{j}^{\prime} \boldsymbol{\theta}\right\}=\mathbf{0} .
$$

For convenience of further discussion, let us define: $\forall k \in\{1, \ldots, \mathbb{K}\}$,

$$
\widehat{\Delta}_{k}\left(\mathbf{x} ; \mathbf{P}_{r}, \widehat{\mathbf{P}}_{r, k}\right)=\widehat{m}_{k}\left(\mathbf{x} ; \widehat{\mathbf{P}}_{r, k}\right)-m\left(\mathbf{x} ; \mathbf{P}_{r}\right) \quad \forall \mathbf{x} \in \mathcal{X}
$$


and

$$
\widehat{\mathbf{G}}_{k}(\mathbf{x})=\overrightarrow{\mathbf{x}} \widehat{\Delta}_{k}\left(\mathbf{x} ; \mathbf{P}_{r}, \widehat{\mathbf{P}}_{r, k}\right)-\mathbb{E}_{\mathbf{X}}\left\{\overrightarrow{\mathbf{X}} \widehat{\Delta}_{k}\left(\mathbf{X} ; \mathbf{P}_{r}, \widehat{\mathbf{P}}_{r, k}\right)\right\} \quad \forall \mathbf{x} \in \mathcal{X},
$$

where $\mathbb{E}_{\mathbf{X}}(\cdot)$ denotes expectation w.r.t. $\mathbf{X} \in \mathcal{U}$. The dependence of $\widehat{\mathbf{G}}_{k}(\cdot)$ on $\left(\mathbf{P}_{r}, \widehat{\mathbf{P}}_{r, k}\right)$ and $\mathbb{P}_{\mathbf{X}}$ is suppressed here for notational simplicity. We now present our main result summarizing the properties of $\widehat{\boldsymbol{\theta}}_{\left(\mathbf{P}_{r}, \mathbb{K}\right)}$.

TheOREM 3.2. Suppose that $\mathcal{T}$ satisfies: (i) $\sup _{\mathbf{x} \in \mathcal{X}}\left|m\left(\mathbf{x} ; \mathbf{P}_{r}\right)\right|<\infty$ and (ii) $\sup _{\mathbf{x} \in \mathcal{X}}\left|\widehat{m}\left(\mathbf{x} ; \widehat{\mathbf{P}}_{r}\right)-m\left(\mathbf{x} ; \mathbf{P}_{r}\right)\right|=O_{p}\left(c_{n}\right)$ for some $c_{n}=o(1)$. With $\widehat{\mathbf{G}}_{k}(\cdot)$ as in (3.9), define $\mathbb{G}_{n, \mathbb{K}}=n^{-\frac{1}{2}} \sum_{k=1}^{\mathbb{K}} \sum_{i \in \mathcal{I}_{k}} \widehat{\mathbf{G}}_{k}\left(\mathbf{X}_{i}\right)$. Then, for any $\mathbb{K} \geq 1$,

$$
n^{\frac{1}{2}}\left(\widehat{\boldsymbol{\theta}}_{\left(\mathbf{P}_{r}, \mathbb{K}\right)}-\boldsymbol{\theta}_{0}\right)=n^{-\frac{1}{2}} \sum_{i=1}^{n} \boldsymbol{\psi}\left(\mathbf{Z}_{i} ; \mathbf{P}_{r}\right)-\boldsymbol{\Gamma}^{-1} \mathbb{G}_{n, \mathbb{K}}+O_{p}\left(c_{n, \mathbb{K}}^{*}\right),
$$

where $\boldsymbol{\psi}\left(\mathbf{Z} ; \mathbf{P}_{r}\right)=\Gamma^{-1}\left[\overrightarrow{\mathbf{X}}\left\{Y-\mu\left(\mathbf{X} ; \mathbf{P}_{r}\right)\right\}\right]$ and $c_{n, \mathbb{K}}^{*}=c_{n_{\mathbb{K}}}+n^{-\frac{1}{2}}+(n / N)^{\frac{1}{2}}=$ $o(1)$. Further, for any fixed $\mathbb{K} \geq 2, \mathbb{G}_{n, \mathbb{K}}=O_{p}\left(c_{n_{\mathbb{K}}^{-}}\right)$, so that

$$
n^{\frac{1}{2}}\left(\widehat{\boldsymbol{\theta}}_{\left(\mathbf{P}_{r}, \mathbb{K}\right)}-\boldsymbol{\theta}_{0}\right)=n^{-\frac{1}{2}} \sum_{i=1}^{n} \boldsymbol{\psi}\left(\mathbf{Z}_{i} ; \mathbf{P}_{r}\right)+O_{p}\left(c_{n_{\mathbb{K}}^{-}}+c_{n, \mathbb{K}}^{*}\right),
$$

which converges in distribution to $\mathcal{N}_{(p+1)}\left[\mathbf{0}, \mathbf{\Sigma}\left\{\mu\left(\cdot ; \mathbf{P}_{r}\right)\right\}\right]$.

REMARK 3.3. If the imputation is "sufficient" so that $\mu\left(\mathbf{x} ; \mathbf{P}_{r}\right)=m(\mathbf{x})$, then $\widehat{\boldsymbol{\theta}}_{\left(\mathbf{P}_{r}, \mathbb{K}\right)}$, for any $\mathbb{K} \geq 2$, enjoys the same set of optimality properties as those noted in Remark 3.1 for $\widehat{\boldsymbol{\theta}}_{n p}$ [while requiring less stringent assumptions about $K(\cdot)$ and $h$, if $\mathrm{KS}$ is used]. If $\mu\left(\mathbf{x} ; \mathbf{P}_{r}\right) \neq m(\mathbf{x})$, then it is however unclear whether $\widehat{\boldsymbol{\theta}}_{\left(\mathbf{P}_{r}, \mathbb{K}\right)}$ is always more efficient than $\widehat{\boldsymbol{\theta}}$. This will be addressed in Section 3.3 where we develop the final EASE.

REMARK 3.4. Apart from the fairly mild condition (i), Theorem 3.2 only requires uniform consistency of $\widehat{m}\left(\cdot ; \widehat{\mathbf{P}}_{r}\right)$ w.r.t. $m\left(\cdot ; \mathbf{P}_{r}\right)$ for establishing the $n^{\frac{1}{2}}$ consistency and asymptotic normality $(\mathrm{CAN})$ of $\widehat{\boldsymbol{\theta}}_{\left(\mathbf{P}_{r}, \mathbb{K}\right)}$ for any $\mathbb{K} \geq 2$. The uniform consistency typically holds for a wide range of smoothing methods $\mathcal{T}$ under fairly general conditions. For $\mathcal{T}:=\mathrm{KS}$ in particular, we provide explicit results in Section 4 under mild regularity conditions that allow the use of any kernel order and the associated optimal bandwidth order. This is a notable relaxation from the stringent requirements for Theorem 3.1 that necessitate under-smoothing and the use of higher order kernels.

REMARK 3.5. The CAN property of $\widehat{\boldsymbol{\theta}}_{\left(\mathbf{P}_{r}, 1\right)}$ has not yet been established. The term $\mathbb{G}_{n, \mathbb{K}}$ in (3.10) behaves quite differently when $\mathbb{K}=1$, compared to $\mathbb{K} \geq 2$ when it has a nice structure due to the inherent "cross-fitting" involved and can 
be controlled easily, and quite generally, under mild conditions as noted in Remark 3.4. For $\mathbb{K}=1$, however, $\mathbb{G}_{n, \mathbb{K}}$ is simply a centered empirical process devoid of any such structure and in general, controlling it requires stronger conditions and the use of empirical process theory. In Section 4, we derive the properties of $\widehat{\boldsymbol{\theta}}_{\left(\mathbf{P}_{r}, 1\right)}$ for the case of $\mathcal{T}:=\mathrm{KS}$ in Theorem 4.2 using a slightly different approach, specialized for KS estimators, to control $\mathbb{G}_{n, 1}$. The main technical tools involved therein are Lemmas A. 2 and A. 3 which may themselves be of independent interest.

3.3. Efficient and adaptive semi-supervised estimators (EASE). To ensure adaptivity even when $\mu\left(\mathbf{x} ; \mathbf{P}_{r}\right) \neq m(\mathbf{x})$, we now define the final EASE as an optimal linear combination of $\widehat{\boldsymbol{\theta}}$ and $\widehat{\boldsymbol{\theta}}_{\left(\mathbf{P}_{\zeta}, \mathbb{K}\right)}$. Specifically, for any fixed $(p+1) \times(p+$ 1) matrix $\boldsymbol{\Delta}, \widehat{\boldsymbol{\theta}}_{\left(\mathbf{P}_{r}, \mathbb{K}\right)}(\boldsymbol{\Delta})=\widehat{\boldsymbol{\theta}}+\boldsymbol{\Delta}\left(\boldsymbol{\boldsymbol { \theta }}_{\left(\mathbf{P}_{r}, \mathbb{K}\right)}-\widehat{\boldsymbol{\theta}}\right)$ is a CAN estimator of $\boldsymbol{\theta}_{0}$ whenever $\widehat{\boldsymbol{\theta}}$ and $\widehat{\boldsymbol{\theta}}_{\left(\mathbf{P}_{r}, \mathbb{K}\right)}$ are, and an optimal $\boldsymbol{\Delta}$ can be selected easily to minimize the asymptotic variance of the combined estimator. For simplicity, we focus here on $\boldsymbol{\Delta}$ being a diagonal matrix with $\boldsymbol{\Delta}=\operatorname{diag}\left(\delta_{1}, \ldots, \delta_{p+1}\right)$. Then the EASE is defined as $\widehat{\boldsymbol{\theta}}_{\left(\mathbf{P}_{r}, \mathbb{K}\right)}^{E} \equiv \widehat{\boldsymbol{\theta}}_{\left(\mathbf{P}_{r}, \mathbb{K}\right)}(\widehat{\boldsymbol{\Delta}})$ with $\widehat{\boldsymbol{\Delta}}$ being any consistent estimator (see Section 3.4 for details) of the minimizer $\overline{\boldsymbol{\Delta}}=\operatorname{diag}\left(\bar{\delta}_{1}, \ldots, \bar{\delta}_{p+1}\right)$, where $\forall 1 \leq l \leq(p+1)$,

$$
\bar{\delta}_{l}=-\lim _{\varepsilon \downarrow 0} \frac{\operatorname{Cov}\left\{\boldsymbol{\psi}_{0[l]}(\mathbf{Z}), \boldsymbol{\psi}_{[l]}\left(\mathbf{Z} ; \mathbf{P}_{r}\right)-\boldsymbol{\psi}_{0[l]}(\mathbf{Z})\right\}}{\operatorname{Var}\left\{\boldsymbol{\psi}_{[l]}\left(\mathbf{Z} ; \mathbf{P}_{r}\right)-\boldsymbol{\psi}_{0[l]}(\mathbf{Z})\right\}+\varepsilon}
$$

and for any vector $\mathbf{a}, \mathbf{a}_{[l]}$ denotes its $l$ th component. Note that in (3.12), the $\varepsilon$ and the limit outside are included to formally account for the case: $\psi_{0[l]}(\mathbf{Z})=$ $\boldsymbol{\psi}_{[l]}\left(\mathbf{Z}, \mathbf{P}_{r}\right)$ a.s. $\left[\mathbb{P}_{\mathbf{Z}}\right]$, when we define $\bar{\delta}_{l}=0$ for identifiability.

It is straightforward to show that $\widehat{\boldsymbol{\theta}}_{\left(\mathbf{P}_{r}, \mathbb{K}\right)}^{E}$ and $\widehat{\boldsymbol{\theta}}_{\left(\mathbf{P}_{r}, \mathbb{K}\right)}(\overline{\boldsymbol{\Delta}})$ are asymptotically equivalent, so that $\widehat{\boldsymbol{\theta}}_{\left(\mathbf{P}_{r}, \mathbb{K}\right)}^{E}$ is a RAL estimator of $\boldsymbol{\theta}_{0}$ satisfying:

$$
\left.n^{\frac{1}{2}} \widehat{\boldsymbol{\theta}}_{\left(\mathbf{P}_{r}, \mathbb{K}\right)}^{E}-\boldsymbol{\theta}_{0}\right)=n^{-\frac{1}{2}} \sum_{i=1}^{n} \boldsymbol{\psi}\left(\mathbf{Z}_{i} ; \mathbf{P}_{r}, \overline{\boldsymbol{\Delta}}\right)+o_{p}(1) \stackrel{d}{\rightarrow} \mathcal{N}_{(p+1)}\left[\mathbf{0}, \Sigma_{\mathbf{P}_{r}}(\overline{\boldsymbol{\Delta}})\right],
$$

as $n \rightarrow \infty$, where $\boldsymbol{\psi}\left(\mathbf{Z} ; \mathbf{P}_{r}, \overline{\boldsymbol{\Delta}}\right)=\boldsymbol{\psi}_{0}(\mathbf{Z})+\overline{\boldsymbol{\Delta}}\left\{\boldsymbol{\psi}\left(\mathbf{Z} ; \mathbf{P}_{r}\right)-\boldsymbol{\psi}_{0}(\mathbf{Z})\right\}$ and $\boldsymbol{\Sigma}_{\mathbf{P}_{r}}(\overline{\mathbf{\Delta}})=$ $\operatorname{Var}\left\{\boldsymbol{\psi}\left(\mathbf{Z} ; \mathbf{P}_{r}, \overline{\boldsymbol{\Delta}}\right)\right\}$. Note that when either the linear model holds or the SNP imputation is sufficient, then $\boldsymbol{\psi}\left(\mathbf{Z} ; \mathbf{P}_{r}, \overline{\boldsymbol{\Delta}}\right)=\boldsymbol{\psi}_{\text {eff }}(\mathbf{Z})$, so that $\widehat{\boldsymbol{\theta}}_{\left(\mathbf{P}_{r}, \mathbb{K}\right)}^{E}$ is asymptotically optimal in the sense of Remark 3.1. Further, when neither cases hold, $\widehat{\boldsymbol{\theta}}_{\left(\mathbf{P}_{r}, \mathbb{K}\right)}^{E}$ is no longer optimal, but is still efficient and adaptive compared to $\widehat{\boldsymbol{\theta}}$. Lastly, if the imputation is certain to be sufficient (e.g., if $r=p$ and $\mathcal{T}:=\mathrm{KS}$ ), we may simply define $\widehat{\boldsymbol{\theta}}_{\left(\mathbf{P}_{r}, \mathbb{K}\right)}^{E}=\widehat{\boldsymbol{\theta}}_{\left(\mathbf{P}_{r}, \mathbb{K}\right)}$.

REMARK 3.6. It can be shown that under $\mathcal{M}_{\mathbf{X}}$, defined in Remark 3.1, the class of all possible IFs achievable by RAL estimators of $\boldsymbol{\theta}_{0}$ is given by: $\mathcal{I F}_{\boldsymbol{\theta}_{0}, \mathcal{M}_{\mathbf{X}}}=\left\{\boldsymbol{\psi}_{\mathbf{g}}(\mathbf{Z}) \equiv \boldsymbol{\psi}_{\mathrm{eff}}(\mathbf{Z})+\mathbf{g}(\mathbf{X}): \mathbb{E}\{\mathbf{g}(\mathbf{X})\}=\mathbf{0}, \mathbf{g}_{[j]}(\cdot) \in \mathcal{L}_{2}\left(\mathbb{P}_{\mathbf{X}}\right) \forall j\right\}$. The 
IFs achieved by $\widehat{\boldsymbol{\theta}}, \widehat{\boldsymbol{\theta}}_{\left(\mathbf{P}_{r}, \mathbb{K}\right)}$ and $\widehat{\boldsymbol{\theta}}_{\left(\mathbf{P}_{r}, \mathbb{K}\right)}^{E}$ are clearly members of this class. The SNP imputation, for various choices of the imputation function $\mu\left(\cdot ; \mathbf{P}_{r}\right)$, therefore, equips us with a family of RAL estimator pairs $\left\{\widehat{\boldsymbol{\theta}}_{\left(\mathbf{P}_{r}, \mathbb{K}\right)}, \widehat{\boldsymbol{\theta}}_{\left(\mathbf{P}_{r}, \mathbb{K}\right)}^{E}\right\}$ for estimating $\boldsymbol{\theta}_{0}$. The IF of $\widehat{\boldsymbol{\theta}}_{\left(\mathbf{P}_{r}, \mathbb{K}\right)}^{E}$ is further guaranteed to dominate that of $\widehat{\boldsymbol{\theta}}$, and when $\mu\left(\cdot ; \mathbf{P}_{r}\right)=m(\cdot)$, it also dominates all other IFs $\in \mathcal{I F}_{\boldsymbol{\theta}_{0}, M_{\mathbf{X}}}$.

3.4. Inference for EASE and the SNP imputation based SS estimators. We now provide procedures for making inference about $\boldsymbol{\theta}_{0}$ based on $\widehat{\boldsymbol{\theta}}_{\left(\mathbf{P}_{r}, \mathbb{K}\right)}$ and $\widehat{\boldsymbol{\theta}}_{\left(\mathbf{P}_{r}, \mathbb{K}\right)}^{E}$ obtained using $\mathbb{K} \geq 2$. We also employ a "double" CV to overcome bias in variance estimation due to over-fitting. A key step involved in the variance estimation is to obtain reasonable estimates of $\left\{\mu\left(\mathbf{X}_{i} ; \mathbf{P}_{r}\right)\right\}_{i=1}^{n}$. Although $\widehat{\boldsymbol{\eta}}_{\left(\mathbf{P}_{r}, \mathbb{K}\right)}$ in (3.4) was constructed via $\mathrm{CV}$, the corresponding estimate, $\widehat{\mu}\left(\mathbf{x} ; \widehat{\mathcal{P}}_{r, \mathbb{K}}\right)$ in (3.6), of $\mu\left(\mathbf{x} ; \mathbf{P}_{r}\right)$ is likely to be over-fitted for $\mathbf{X}_{i} \in \mathcal{L}$. To construct bias corrected estimates of $\mu\left(\mathbf{X}_{i} ; \mathbf{P}_{r}\right)$, we first obtain $\mathbb{K}$ separate doubly cross-validated estimates of $\eta_{\mathbf{P}_{r}},\left\{\widehat{\boldsymbol{\eta}}_{\left(\mathbf{P}_{r}, \mathbb{K}\right)}^{k}: k=1, \ldots, \mathbb{K}\right\}$, with $\widehat{\boldsymbol{\eta}}_{\left(\mathbf{P}_{r}, \mathbb{K}\right)}^{k}$, for each $k$, being the solution in $\boldsymbol{\eta}$ to $\sum_{k^{\prime} \neq k} \mathcal{S}_{k^{\prime}}(\boldsymbol{\eta})=\mathbf{0}$, where

$$
\mathcal{S}_{k^{\prime}}(\boldsymbol{\eta})=\sum_{i \in \mathcal{I}_{k^{\prime}}} \overrightarrow{\mathbf{X}}_{i}\left\{Y_{i}-\widehat{m}_{k^{\prime}}\left(\mathbf{X}_{i} ; \widehat{\mathbf{P}}_{r, k^{\prime}}\right)-\overrightarrow{\mathbf{X}}_{i}^{\prime} \boldsymbol{\eta}\right\} \quad \forall k^{\prime} \in\{1, \ldots, \mathbb{K}\} .
$$

For each $k$ and $k^{\prime} \neq k, \mathcal{S}_{k^{\prime}}(\boldsymbol{\eta})$ is constructed such that $\left\{\mathbf{Z}_{i}: i \in \mathcal{I}_{k^{\prime}}\right\}$ used for obtaining $\widehat{\boldsymbol{\eta}}_{\left(\mathbf{P}_{r}, \mathbb{K}\right)}^{k}$ is independent of $\widehat{m}_{k^{\prime}}\left(\cdot ; \widehat{\mathbf{P}}_{r, k^{\prime}}\right)$ that is based on $\mathcal{L}_{k^{\prime}}^{-} \Perp \mathcal{L}_{k^{\prime}}$. Then, for each $\mathbf{X}_{i} \in \mathcal{L}_{k}$ and $k \in\{1, \ldots, \mathbb{K}\}$, we may estimate $\mu\left(\mathbf{X}_{i} ; \mathbf{P}_{r}\right)$ as

$$
\widehat{\mu}_{k}\left(\mathbf{X}_{i} ; \widehat{\mathcal{P}}_{r, \mathbb{K}}\right)=\widehat{m}_{k}\left(\mathbf{X}_{i} ; \widehat{\mathbf{P}}_{r, k}\right)+\overrightarrow{\mathbf{X}}_{i}^{\prime} \widehat{\boldsymbol{\eta}}_{\left(\mathbf{P}_{r}, \mathbb{K}\right)}^{k} .
$$

We exclude $\mathcal{S}_{k}(\boldsymbol{\eta})$ in the construction of $\widehat{\boldsymbol{\eta}}_{\left(\mathbf{P}_{r}, \mathbb{K}\right)}^{k}$ to reduce over-fitting bias in the residuals $\left\{Y_{i}-\widehat{\mu}_{k}\left(\mathbf{X}_{i} ; \widehat{\mathcal{P}}_{r, \mathbb{K}}\right)\right\}$, which we now use for estimating the IFs.

For each $\mathbf{Z}_{i} \in \mathcal{L}_{k}$ and $k \in\{1, \ldots, \mathbb{K}\}$, we estimate $\boldsymbol{\psi}_{0}\left(\mathbf{Z}_{i}\right)$ and $\boldsymbol{\psi}\left(\mathbf{Z}_{i} ; \mathbf{P}_{r}\right)$, the corresponding IFs of $\widehat{\boldsymbol{\theta}}$ and $\widehat{\boldsymbol{\theta}}_{\left(\mathbf{P}_{r}, \mathbb{K}\right)}$, respectively, as

$$
\begin{aligned}
\widehat{\boldsymbol{\psi}}_{0}\left(\mathbf{Z}_{i}\right) & =\widehat{\boldsymbol{\Gamma}}^{-1}\left\{\overrightarrow{\mathbf{X}}_{i}\left(Y_{i}-\overrightarrow{\mathbf{X}}_{i}^{\prime} \widehat{\boldsymbol{\theta}}\right)\right\} \quad \text { and } \\
\widehat{\boldsymbol{\psi}}_{k}\left(\mathbf{Z}_{i} ; \mathbf{P}_{r}\right) & =\widehat{\boldsymbol{\Gamma}}^{-1}\left[\overrightarrow{\mathbf{X}}_{i}\left\{Y_{i}-\widehat{\mu}_{k}\left(\mathbf{X}_{i} ; \widehat{\mathcal{P}}_{r, \mathbb{K}}\right)\right\}\right],
\end{aligned}
$$

where $\widehat{\boldsymbol{\Gamma}}$ denotes any consistent estimator of $\boldsymbol{\Gamma}$ from $\mathcal{L}$ and/or $\mathcal{U}$ (e.g., $\widehat{\boldsymbol{\Gamma}}=\boldsymbol{\Gamma}_{n} \equiv$ $n^{-1} \sum_{i=1}^{n} \overrightarrow{\mathbf{X}}_{i} \overrightarrow{\mathbf{X}}_{i}^{\prime}$ based on $\mathcal{L}$, or $\widehat{\boldsymbol{\Gamma}}=\boldsymbol{\Gamma}_{N} \equiv N^{-1} \sum_{j=n+1}^{n+N} \overrightarrow{\mathbf{X}}_{j} \overrightarrow{\mathbf{X}}_{j}^{\prime}$ based on $\mathcal{U}$ ). Then, $\boldsymbol{\Sigma}\left\{\mu\left(\cdot ; \mathbf{P}_{r}\right)\right\}$ in (3.11) may be consistently estimated as

$$
\widehat{\boldsymbol{\Sigma}}\left\{\mu\left(\cdot ; \mathbf{P}_{r}\right)\right\}=n^{-1} \sum_{k=1}^{\mathbb{K}} \sum_{i \in \mathcal{I}_{k}} \widehat{\boldsymbol{\psi}}_{k}\left(\mathbf{Z}_{i} ; \mathbf{P}_{r}\right) \widehat{\boldsymbol{\psi}}_{k}^{\prime}\left(\mathbf{Z}_{i} ; \mathbf{P}_{r}\right) .
$$


To estimate the combination matrix $\overline{\boldsymbol{\Delta}}$ in (3.12) and the asymptotic variance, $\boldsymbol{\Sigma}_{\mathbf{P}_{r}}(\overline{\boldsymbol{\Delta}})$, of EASE consistently, let us define, $\forall 1 \leq l \leq(p+1)$,

$$
\begin{aligned}
& \widehat{\sigma}_{l, 12}=-n^{-1} \sum_{k=1}^{\mathbb{K}} \sum_{i \in \mathcal{I}_{k}} \widehat{\boldsymbol{\psi}}_{0[l]}\left(\mathbf{Z}_{i}\right)\left\{\widehat{\boldsymbol{\psi}}_{k[l]}\left(\mathbf{Z}_{i} ; \mathbf{P}_{r}\right)-\widehat{\boldsymbol{\psi}}_{0[l]}\left(\mathbf{Z}_{i}\right)\right\} \\
& \widehat{\sigma}_{l, 22}=n^{-1} \sum_{k=1}^{\mathbb{K}} \sum_{i \in \mathcal{I}_{k}}\left\{\widehat{\boldsymbol{\psi}}_{k[l]}\left(\mathbf{Z}_{i} ; \mathbf{P}_{r}\right)-\widehat{\boldsymbol{\psi}}_{0[l]}\left(\mathbf{Z}_{i}\right)\right\}^{2}
\end{aligned}
$$

and $\widehat{\delta}_{l}=\widehat{\sigma}_{l, 12} /\left(\widehat{\sigma}_{l, 22}+\varepsilon_{n}\right)$ for some sequence $\varepsilon_{n} \rightarrow 0$ with $n^{\frac{1}{2}} \varepsilon_{n} \rightarrow \infty$. Then we estimate $\overline{\boldsymbol{\Delta}}$ and $\boldsymbol{\Sigma}_{\mathbf{P}_{r}}(\overline{\boldsymbol{\Delta}})$, respectively, as $\widehat{\boldsymbol{\Delta}}=\operatorname{diag}\left(\widehat{\delta}_{1}, \ldots, \widehat{\delta}_{p+1}\right)$ and

$$
\widehat{\boldsymbol{\Sigma}}_{\mathbf{P}_{r}}(\widehat{\boldsymbol{\Delta}})=n^{-1} \sum_{k=1}^{\mathbb{K}} \sum_{i \in \mathcal{I}_{k}} \widehat{\boldsymbol{\psi}}_{k}\left(\mathbf{Z}_{i} ; \mathbf{P}_{r}, \widehat{\boldsymbol{\Delta}}\right) \widehat{\boldsymbol{\psi}}_{k}^{\prime}\left(\mathbf{Z}_{i} ; \mathbf{P}_{r}, \widehat{\boldsymbol{\Delta}}\right)
$$

where $\widehat{\boldsymbol{\psi}}_{k}\left(\mathbf{Z} ; \mathbf{P}_{r}, \widehat{\boldsymbol{\Delta}}\right)=\widehat{\boldsymbol{\psi}}_{0}(\mathbf{Z})+\widehat{\boldsymbol{\Delta}}\left\{\widehat{\boldsymbol{\psi}}_{k}\left(\mathbf{Z} ; \mathbf{P}_{r}\right)-\widehat{\boldsymbol{\psi}}_{0}(\mathbf{Z})\right\} \forall k \in\{1, \ldots, \mathbb{K}\}$. Normal confidence intervals (CIs) for the parameters of interest can also be constructed accordingly based on these variance estimates.

4. Implementation based on KS. We next detail the specific implementation of the SNP imputation based on KS estimators. With $\mathcal{T}:=\mathrm{KS}$, the target function for the smoothing is given by: $m\left(\mathbf{x} ; \mathbf{P}_{r}\right)=m_{\mathbf{P}_{r}}\left(\mathbf{P}_{r}^{\prime} \mathbf{x}\right) \equiv \mathbb{E}\left(Y \mid \mathbf{X}_{\mathbf{P}_{r}}=\mathbf{P}_{r}^{\prime} \mathbf{x}\right)$. For simplicity, we assume that $\mathbf{X}_{\mathbf{P}_{r}}$ is continuous with a density $f_{\mathbf{P}_{r}}(\cdot)$ and support $\mathcal{X}_{\mathbf{P}_{r}} \equiv\left\{\mathbf{P}_{r}^{\prime} \mathbf{x}: \mathbf{x} \in \mathcal{X}\right\} \subseteq \mathbb{R}^{r}$. Let us now consider the following class of local constant KS estimators for $m\left(\mathbf{x} ; \mathbf{P}_{r}\right)$ :

$$
\widehat{m}_{k}\left(\mathbf{x} ; \widehat{\mathbf{P}}_{r, k}\right)=\frac{\frac{1}{n_{\mathbb{K}}^{-} h^{r}} \sum_{i \in \mathcal{I}_{k}^{-}}\left\{K_{h}\left(\widehat{\mathbf{P}}_{r, k}^{\prime} \mathbf{X}_{i}, \widehat{\mathbf{P}}_{r, k}^{\prime} \mathbf{x}\right)\right\} Y_{i}}{\frac{1}{n_{\mathbb{K}}^{-} h^{r}} \sum_{i \in \mathcal{I}_{k}^{-}} K_{h}\left(\widehat{\mathbf{P}}_{r, k}^{\prime} \mathbf{X}_{i}, \widehat{\mathbf{P}}_{r, k}^{\prime} \mathbf{x}\right)} \quad \forall 1 \leq k \leq \mathbb{K},
$$

where $K_{h}(\cdot)$ and $h$ are as in Section 3.1 with $K(\cdot)$ now being a suitable kernel on $\mathbb{R}^{r}$. In the light of Theorem 3.2, we focus primarily on establishing the uniform consistency of $\widehat{m}\left(\mathbf{x} ; \widehat{\mathbf{P}}_{r}\right) \equiv \widehat{m}_{1}\left(\mathbf{x} ; \widehat{\mathbf{P}}_{r, 1}\right)$ in (4.1) with $\mathbb{K}=1$, accounting for the additional estimation error from $\widehat{\mathbf{P}}_{r}$. For establishing the desired result, we shall assume the following regularity conditions to hold.

AsSUMPTION 4.1. (i) $K(\cdot)$ is a symmetric kernel of order $q \geq 2$ with finite $q$ th moments. (ii) $K(\cdot)$ is bounded, integrable and is either Lipschitz continuous with a compact support or, has a bounded derivative $\nabla K(\cdot)$ which satisfies: $\|\nabla K(\mathbf{z})\| \leq \Lambda\|\mathbf{z}\|^{-\rho} \forall \mathbf{z} \in \mathbb{R}^{r}$ with $\|\mathbf{z}\|>L$, where $\Lambda>0, L>0$ and $\rho>1$ are some fixed constants, and $\|\cdot\|$ denotes the standard $L_{2}$ vector norm. (iii) $\mathcal{X}_{\mathbf{P}_{r}} \subseteq \mathbb{R}^{r}$ is compact. $\mathbb{E}\left(|Y|^{s}\right)<\infty$ for some $s>2$. $\mathbb{E}\left(|Y|^{s} \mid \mathbf{X}_{\mathbf{P}_{r}}=\mathbf{z}\right) f_{\mathbf{P}_{r}}(\mathbf{z})$ and $f_{\mathbf{P}_{r}}(\mathbf{z})$ are bounded on $\mathcal{X}_{\mathbf{P}_{r}}$. (iv) $f_{\mathbf{P}_{r}}(\mathbf{z})$ is bounded away from 0 on $\mathcal{X}_{\mathbf{P}_{r}}$. (v) $m_{\mathbf{P}_{r}}(\mathbf{z})$ and 
$f_{\mathbf{P}_{r}}(\mathbf{z})$ are both $q$ times continuously differentiable with bounded $q$ th derivatives on some open set $\mathcal{X}_{0, \mathbf{P}_{r}} \supseteq \mathcal{X}_{\mathbf{P}_{r}}$. Additional conditions (required only when $\mathbf{P}_{r}$ needs to be estimated): (vi) $K(\cdot)$ has a bounded and integrable derivative $\nabla K(\cdot)$. (vii) $\nabla K(\cdot)$ satisfies: $\left\|\nabla K\left(\mathbf{z}_{1}\right)-\nabla K\left(\mathbf{z}_{2}\right)\right\| \leq\left\|\mathbf{z}_{1}-\mathbf{z}_{2}\right\| \phi\left(\mathbf{z}_{1}\right) \forall \mathbf{z}_{1}, \mathbf{z}_{2} \in \mathbb{R}^{r}$ such that $\left\|\mathbf{z}_{1}-\mathbf{z}_{2}\right\| \leq L^{*}$, for some fixed constant $L^{*}>0$, and some bounded and integrable function $\phi: \mathbb{R}^{r} \rightarrow \mathbb{R}^{+}$. (viii) $\nabla K(\cdot)$ is Lipschitz continuous on $\mathbb{R}^{r}$. (ix) $\mathbb{E}\left(\mathbf{X} \mid \mathbf{X}_{\mathbf{P}_{r}}=\mathbf{z}\right)$ and $\mathbb{E}\left(\mathbf{X} Y \mid \mathbf{X}_{\mathbf{P}_{r}}=\mathbf{z}\right)$ are both continuously differentiable with bounded first derivatives on $\mathcal{X}_{0, \mathbf{P}_{r}} \supseteq \mathcal{X}_{\mathbf{P}_{r}}$.

Assumption 4.1, mostly adopted from Hansen (2008), imposes some mild smoothness and moment conditions most of which are fairly standard, except perhaps the conditions on $K(\cdot)$ in (vi)-(viii) all of which are however satisfied by the Gaussian kernel among others. We now propose the following result.

THEOREM 4.1. Suppose $\left(\widehat{\mathbf{P}}_{r}-\mathbf{P}_{r}\right)=O_{p}\left(\alpha_{n}\right)$ for some $\alpha_{n}=o(1)$ with $\alpha_{n}=0$ identically if $\mathbf{P}_{r}$ is known. Let $q$ be the order of the kernel $K(\cdot)$ in (4.1) for some integer $q \geq 2$. Define

$$
a_{n, 1}=\alpha_{n}\left(\frac{\log n}{n h^{r+2}}\right)^{\frac{1}{2}}+\alpha_{n}^{2} h^{-(r+2)}+\alpha_{n}, \quad a_{n, 2}=\left(\frac{\log n}{n h^{r}}\right)^{\frac{1}{2}}+h^{q}
$$

and assume that each of the terms involved in $a_{n, 1}=o(1)$ and $a_{n, 2}=o(1)$. Then, under Assumption 4.1, $\widehat{m}\left(\mathbf{x} ; \widehat{\mathbf{P}}_{r}\right)$, based on (4.1), satisfies

$$
\sup _{\mathbf{x} \in \mathcal{X}}\left|\widehat{m}\left(\mathbf{x} ; \widehat{\mathbf{P}}_{r}\right)-m\left(\mathbf{x} ; \mathbf{P}_{r}\right)\right|=O_{p}\left(a_{n, 1}+a_{n, 2}\right) .
$$

REMARK 4.1. Theorem 4.1 establishes the $L_{\infty}$ error rate of $\widehat{m}\left(\mathbf{x} ; \widehat{\mathbf{P}}_{r}\right)$ under mild regularity conditions and restrictions on $h$. Among its various implications, the rate also ensures uniform consistency of $\widehat{m}\left(\mathbf{x} ; \widehat{\mathbf{P}}_{r}\right)$ at the optimal bandwidth order: $h_{\mathrm{opt}}=O\left(n^{-1 /(2 q+r)}\right)$ for any kernel order $q \geq 2$ and any $r \leq p$, as long as $\alpha_{n}=o\left(n^{-(r+2) /(4 q+2 r)}\right)$ which always includes: $\alpha_{n}=O\left(n^{-\frac{1}{2}}\right)$ and $\alpha_{n}=0$. These two cases are particularly relevant in practice as $\mathbf{P}_{r}$ being finite dimensional, $n^{\frac{1}{2}}$ consistent estimators of $\mathbf{P}_{r}$ should typically exist. For both cases, using $h_{\text {opt }}$ results in $a_{n, 1}$ to be of lower order (for $q>2$ ) or the same order (for $q=2$ ) compared to that of the main term $a_{n, 2}$, so that the usual optimal rate prevails as the overall error rate.

Properties of $\widehat{\boldsymbol{\theta}}_{\left(\mathbf{P}_{r}, \mathbb{K}\right)}$ for $\mathbb{K}=1$. We now address the CAN property of $\widehat{\boldsymbol{\theta}}_{\left(\mathbf{P}_{r}, \mathbb{K}\right)}$ for $\mathbb{K}=1$ under the KS framework. Based on (3.10) and Remark 3.5, the only step required for this is to effectively control the term $\mathbb{G}_{n, \mathbb{K}}$ in (3.10). We propose the following result in this regard. 
THEOREM 4.2. Let $\mathbb{K}=1, \mathcal{T}:=K S, \mathbb{G}_{n, \mathbb{K}}$ be as in $(3.10)$, and $\widehat{m}\left(\mathbf{x} ; \widehat{\mathbf{P}}_{r}\right)$ be the KS estimator based on (4.1). Let $\alpha_{n}, a_{n, 1}$ and $a_{n, 2}$ be as in Theorem 4.1 with $\left(\widehat{\mathbf{P}}_{r}-\mathbf{P}_{r}\right)=O_{p}\left(\alpha_{n}\right)$. Assume that $a_{n, 1}^{*}$ and $a_{n, 2}^{*}$ are o(1), where $a_{n, 1}^{*}=\alpha_{n}+\frac{\alpha_{n}}{n^{\frac{1}{2}} h^{(r+1)}}+n^{\frac{1}{2}} \alpha_{n}^{2} h^{-2}+n^{\frac{1}{2}} a_{n, 1}^{2}+n^{\frac{1}{2}} a_{n, 1} a_{n, 2} \quad$ and $\quad a_{n, 2}^{*}=n^{\frac{1}{2}} a_{n, 2}^{2}$.

Then, under Assumption 4.1, $\mathbb{G}_{n, \mathbb{K}}=O_{p}\left(a_{n, 1}^{*}+a_{n, 2}^{*}\right)=o_{p}(1)$. Further, let $c_{n, \mathbb{K}}^{*}$ be as in Theorem 3.2 with $c_{n}=\left(a_{n, 1}+a_{n, 2}\right)$. Then, using (3.10),

$$
n^{\frac{1}{2}}\left(\widehat{\boldsymbol{\theta}}_{\left(\mathbf{P}_{r}, \mathbb{K}\right)}-\boldsymbol{\theta}_{0}\right)=n^{-\frac{1}{2}} \sum_{i=1}^{n} \boldsymbol{\psi}\left(\mathbf{Z}_{i}, \mathbf{P}_{r}\right)+O_{p}\left(c_{n, \mathbb{K}}^{*}+d_{n}\right),
$$

where $d_{n}=a_{n, 1}^{*}+a_{n, 2}^{*}$. Hence, $n^{\frac{1}{2}}\left(\widehat{\boldsymbol{\theta}}_{\left(\mathbf{P}_{r}, \mathbb{K}\right)}-\boldsymbol{\theta}_{0}\right) \stackrel{d}{\rightarrow} \mathcal{N}_{(p+1)}\left[\mathbf{0}, \boldsymbol{\Sigma}\left\{\mu\left(\cdot ; \mathbf{P}_{r}\right)\right\}\right]$.

REMARK 4.2. Note that the term $a_{n, 2}^{*}$ always requires $q>r / 2$ in order to converge to 0 , thus showing the contrasting behavior of the case $\mathbb{K}=1$ compared to $\mathbb{K} \geq 2$ where no such higher order kernel restriction is required. Nevertheless, when $\alpha_{n}=O\left(n^{-\frac{1}{2}}\right)$ or $\alpha_{n}=0$, the optimal bandwidth order: $h_{\mathrm{opt}}=O\left(n^{-1 /(2 q+r)}\right)$ can indeed be still used as long as $q>r / 2$ is satisfied. Despite these facts and all the theoretical guarantee in Theorem 4.2, empirical evidence however seems to suggest that $\widehat{\boldsymbol{\theta}}_{\left(\mathbf{P}_{r}, 1\right)}$ can be substantially biased in finite samples, in part due to over-fitting.

REMARK 4.3 (Technical benefits of refitting and CV). Suppose that $\mathbf{P}_{r}=I_{p}$, so that the SNP imputation with $\mathcal{T}:=\mathrm{KS}$ is indeed sufficient. Further, assume that all of Theorems 3.1-4.2 hold, so that the estimators $\widehat{\boldsymbol{\theta}}_{n p}, \widehat{\boldsymbol{\theta}}_{\left(\mathbf{P}_{r}, 1\right)}$ and $\widehat{\boldsymbol{\theta}}_{\left(\mathbf{P}_{r}, \mathbb{K}\right)}(\mathbb{K} \geq 2)$ are comparable and all asymptotically optimal. However, their constructions are quite different which can significantly affect their finite sample performances. $\widehat{\boldsymbol{\theta}}_{n p}$ is based on KS only, and requires stringent under-smoothing and a kernel of order $q>p$ (Remark 3.2); $\widehat{\boldsymbol{\theta}}_{\left(\mathbf{P}_{r}, 1\right)}$ is based on KS and refitting (although the KS itself is certain to be sufficient), and requires no under-smoothing but needs a (weaker) kernel order condition $(q>p / 2)(\operatorname{Remark} 4.2)$; while $\widehat{\boldsymbol{\theta}}_{\left(\mathbf{P}_{r}, \mathbb{K}\right)}(\mathbb{K} \geq 2)$ additionally involves $\mathrm{CV}$, and requires no under-smoothing or higher order kernel conditions (Remark 3.4). This highlights the critical role played by refitting and CV, apart from their primary roles in the SNP imputation, in removing any under-smoothing and/or higher order kernel restrictions when $\mathcal{T}:=\mathrm{KS}$, and this continues to hold for any other $\left(r, \mathbf{P}_{r}\right)$ as well. In particular, it shows, rather surprisingly, that refitting should be performed in order to avoid under-smoothing even if the smoothing is known to be sufficient.

REMARK 4.4. As mentioned in Section 3.2, $\mathcal{T}:=\mathrm{KS}$ along with possible dimension reduction is just one reasonable choice of $\mathcal{T}$ for implementing the SNP 
imputation, all technical requirements for which have been thoroughly established in Section 4. In general, other smoothing methods, as long as the requirements are satisfied, can also be equally used as choices of $\mathcal{T}$. One such choice could be kernel machine $(\mathrm{KM})$ regression (with possibly no use of dimension reduction, as KM uses penalization to effectively regularize the target even with $\mathbf{P}_{r}=I_{p}$ ). We leave its implementation details to the reader as they are readily available in a multitude of references, and also skip any theoretical treatment, considering the primary goal and scope of this paper. However, detailed numerical results are presented in Section 6 for this choice of $\mathcal{T}$ as well to illustrate the wider applicability of our proposed methods.

5. Choices of $\mathbf{P}_{\boldsymbol{r}}$ : Dimension reduction techniques. We next discuss choosing and estimating the matrix $\mathbf{P}_{r}(r<p)$ to be used for dimension reduction, if required, in the SNP imputation, and which can play an important role in the sufficiency of the imputation. Simple choices of $\mathbf{P}_{r}$ include $r$ leading principal component directions of $\mathbf{X}$ or any $r$ canonical directions of $\mathbf{X}$. Note that under the SS setting, $\mathbf{P}_{r}$ is effectively known if it only involves the distribution of $\mathbf{X}$, as is true for these choices. We now focus primarily on the case where $\mathbf{P}_{r}$ also depends on the distribution of $Y$, and hence, is unknown. Such a choice of $\mathbf{P}_{r}$ is often desirable to ensure that the imputation is as "sufficient" as possible for predicting $Y$. Several reasonable choices of such $\mathbf{P}_{r}$ and their estimation are possible based on sufficient dimension reduction (s.d.r.) methods like Sliced Inverse Regression (SIR) [Li (1991)], Principal Hessian Directions (PHD) [Cook (1998), Li (1992)], Sliced Average Variance Estimation (SAVE) [Cook and Lee (1999), Cook and Weisberg (1991)], etc.

In particular, we focus here on SIR where the choice of $\mathbf{P}_{r}$ is given by: $\mathbf{P}_{r}^{0}=$ $\boldsymbol{\Sigma}^{-\frac{1}{2}} \overline{\mathbf{P}}_{r}$, with $\overline{\mathbf{P}}_{r}$ being the $r$ leading eigenvectors of $\mathbb{M}=\operatorname{Var}\{\mathbb{E}(\mathbb{X} \mid Y)\}$, where $\mathbb{X}=\boldsymbol{\Sigma}^{-\frac{1}{2}}(\mathbf{X}-\boldsymbol{\mu})$, with $\boldsymbol{\mu}=\mathbb{E}(\mathbf{X})$, denotes the standardized version of $\mathbf{X}$. It is well known [Li (1991)] that these directions lead to an optimal (in some appropriate sense) $r$-dimensional linear transformation of $\mathbf{X}$ that can be predicted by $Y$. Apart from these general optimality, they also have deeper implications in the context of s.d.r. We refer the reader to [Li (1991)] and other relevant references in the s.d.r. literature for further details.

For estimating $\mathbf{P}_{r}^{0}$, we consider the SIR algorithm of Li (1991) and further propose a SS modification to it. With $\mathbb{K}$ and $\left\{\mathcal{L}_{k}^{-}, \mathcal{I}_{k}^{-}, \widehat{\mathbf{P}}_{r, k}\right\}_{k=1}^{\mathbb{K}}$ as before, let $\left(\widehat{\boldsymbol{\mu}}_{k}, \widehat{\boldsymbol{\Sigma}}_{k}\right)$ denote the estimates of $(\boldsymbol{\mu}, \boldsymbol{\Sigma})$ based on $\mathcal{L}_{k}^{-}$and define $\mathbb{X}^{(k)}=\widehat{\boldsymbol{\Sigma}}_{k}^{-\frac{1}{2}}\left(\mathbf{X}-\widehat{\boldsymbol{\mu}}_{k}\right)$. Then the original SIR algorithm estimates $\mathbf{P}_{r}^{0}$ based on $\mathcal{L}_{k}^{-}$as follows: (i) Divide the range of $\left\{Y_{i}\right\}_{i \in \mathcal{I}_{k}^{-}}$into $H$ slices $\left\{I_{1}, \ldots, I_{H}\right\}$, where $H$ may depend on $n_{\mathbb{K}}^{-}$. For $1 \leq h \leq H$, let $\widehat{p}_{h, k}$ denote the proportion of $\left\{Y_{i}\right\}_{i \in \mathcal{I}_{k}^{-}}$in slice $I_{h}$; (ii) For each $I_{h}$, let $\widehat{\mathbb{M}}_{h, k}$ denote the sample average of the set: $\left\{\mathbb{X}_{i}^{(k)} \in \mathcal{L}_{k}^{-}: Y_{i} \in I_{h}\right\}$; (iii) Estimate $\mathbb{M}$ as: $\widehat{\mathbb{M}}_{k}=\sum_{h=1}^{H} \widehat{p}_{h, k} \widehat{\mathbb{M}}_{h, k} \widehat{\mathbb{M}}_{h, k}^{\prime}$ and $\mathbf{P}_{r}^{0}$ as: $\widehat{\mathbf{P}}_{r, k}^{0}=\widehat{\boldsymbol{\Sigma}}_{k}^{-\frac{1}{2}} \widehat{\mathbf{P}}_{r, k}$, where $\widehat{\mathbf{P}}_{r, k}$ denotes 
the $r$ leading eigenvectors of $\widehat{\mathbb{M}}_{k}$. However, the SIR algorithm often tends to give unstable estimates of $\mathbf{P}_{r}^{0}$, especially for the directions corresponding to the smaller eigenvalues of $\mathbb{M}$. To improve the efficiency in estimating $\mathbf{P}_{r}^{0}$, we now propose a semi-supervised SIR (SS-SIR) algorithm as follows.

SS-SIR algorithm. Given $\left\{\mathcal{L}_{k}^{-}, \mathcal{I}_{k}^{-}, \widehat{\mathbf{P}}_{r, k}\right\}_{k=1}^{\mathbb{K}}$, let $\left(\widehat{\boldsymbol{\mu}}_{k}^{*}, \widehat{\boldsymbol{\Sigma}}_{k}^{*}\right)$ denote the estimates of $(\boldsymbol{\mu}, \boldsymbol{\Sigma})$ based on $\mathcal{L}_{k}^{-} \cup \mathcal{U}$ and define $\mathbb{X}^{(k *)}=\widehat{\boldsymbol{\Sigma}}_{k}^{*-\frac{1}{2}}\left(\mathbf{X}-\widehat{\boldsymbol{\mu}}_{k}^{*}\right)$. Then the SSSIR proceeds as follows. Step (i) stays the same as in SIR. In step (ii), for each $k$, and each $j \in\{n+1, \ldots, n+N\}$, we impute $Y_{j}$ as $Y_{j, k}^{*}=Y_{\widehat{i}_{j, k}}$, where $\widehat{i}_{j, k}=$ $\operatorname{argmin}_{i \in \mathcal{I}_{k}^{-}}\left\|\mathbb{X}_{i}^{(k *)}-\mathbb{X}_{j}^{(k *)}\right\|^{2}$. For each $I_{h}$, let $\widehat{\mathbb{M}}_{h, k}^{*}$ be the sample average of the set: $\left\{\mathbb{X}_{i}^{(k *)} \in \mathcal{L}_{k}^{-}: Y_{i} \in I_{h}\right\} \cup\left\{\mathbb{X}_{j}^{(k *)} \in \mathcal{U}: Y_{j, k}^{*} \in I_{h}\right\}$. Then in step (iii), we estimate $\mathbb{M}$ as: $\widehat{\mathbb{M}}_{k}^{*}=\sum_{h=1}^{H} \widehat{p}_{h, k} \widehat{\mathbb{M}}_{h, k}^{*} \widehat{\mathbb{M}}_{h, k}^{*^{\prime}}$ and then, $\mathbf{P}_{r}^{0}$ as: $\widehat{\mathbf{P}}_{r, k}^{0 *}=\widehat{\boldsymbol{\Sigma}}_{k}^{*-\frac{1}{2}} \widehat{\mathbf{P}}_{r, k}^{*}$, where $\widehat{\mathbf{P}}_{r, k}^{*}$ denotes the $r$ leading eigenvectors of $\widehat{\mathbb{M}}_{k}^{*}$.

The SS-SIR algorithm aims to improve the estimation of $\mathbf{P}_{r}^{0}$ by making use of $\mathcal{U}$ in step (ii) through a nearest neighbour approximation for the unobserved $Y$ in $\mathcal{U}$ using $\mathcal{L}_{k}^{-}$. With $n_{\mathbb{K}}^{-}$large enough and $m(\cdot)$ smooth enough, the imputed and the true underlying $Y$ should belong to the same slice with a high probability. Thus the set of $\mathbb{X}$ 's belonging to a particular slice is now "enriched", and consequently, improved estimation of $\mathbb{M}$ and $\mathbf{P}_{r}^{0}$ is expected. The proposed method based on a nearest neighbor approximation is also highly scalable and while other smoothing based approximations may be used, they can be computationally intensive. The SSSIR algorithm is fairly robust to the choice of $H$, and $H=O\left(n^{\frac{1}{2}} \log n\right)$ seems to give fairly satisfactory performance. The slices may be chosen to have equal width or equal number of observations. For SIR, $n^{\frac{1}{2}}$-consistency of the estimates are well established [Duan and Li (1991), Li (1991), Zhu and Ng (1995)] for various formulations under fairly general settings (without any model based assumptions). The theoretical properties of SS-SIR, although not derived here, are expected to follow similarly. Our simulation results (not shown here) further suggest that SSSIR significantly outperforms SIR, leading to substantially improved estimation of $\boldsymbol{\theta}_{0}$ from the proposed methods.

\section{Numerical studies.}

6.1. Simulation studies. We conducted extensive simulation studies to examine the finite sample performance of our proposed point and interval estimation procedures as well as to compare with existing methods. Throughout, we let $n=500, N=10,000$ and considered $p=2,10$ and 20. For our CV based methods, we let $\mathbb{K}=5$. The true values of the target parameter $\boldsymbol{\theta}_{0}$ were estimated via Monte Carlo with a large sample size of 50,000. For each configuration, the results were summarized based on 500 replications. Results for $p=2$ are summarized in 
the Supplementary Material [Chakrabortty and Cai (2018)], and the discussions below focus primarily on $p=10$ and 20 .

We generated $\mathbf{X} \sim \mathcal{N}_{p}\left[\mathbf{0}, I_{p}\right]$ and restricted $\mathbf{X}$ to $[-5,5]^{p}$ to ensure its boundedness. Given $\mathbf{X}=\mathbf{x}$, we generated $Y \sim \mathcal{N}_{1}[m(\mathbf{x}), 1]$, where we considered four different choices of $m(\mathbf{x})$ :

(i) Linear: $m(\mathbf{x})=\mathbf{x}^{\prime} \mathbf{b}_{p}$;

(ii) Nonlinear one component (NLIC): $m(\mathbf{x})=\left(\mathbf{x}^{\prime} \mathbf{b}_{p}\right)+\left(\mathbf{x}^{\prime} \mathbf{b}_{p}\right)^{2}$;

(iii) Nonlinear two component (NL2C): $m(\mathbf{x})=\left(\mathbf{x}^{\prime} \mathbf{b}_{p}\right)\left(1+\mathbf{x}^{\prime} \boldsymbol{\delta}_{p}\right)$; and

(iv) Nonlinear three component $(N L 3 C): m(\mathbf{x})=\left(\mathbf{x}^{\prime} \mathbf{b}_{p}\right)\left(1+\mathbf{x}^{\prime} \boldsymbol{\delta}_{p}\right)+\left(\mathbf{x}^{\prime} \boldsymbol{\omega}_{p}\right)^{2}$;

where, for each setting, we considered $\mathbf{b}_{p}=\mathbf{b}_{p}^{(1)} \equiv\left(\mathbf{1}_{p / 2}^{\prime}, \mathbf{0}_{p / 2}^{\prime}\right)^{\prime}$ and $\mathbf{b}_{p}=\mathbf{b}_{p}^{(2)} \equiv$ $\mathbf{1}_{p}$, and set $\boldsymbol{\delta}_{p}=\left(\mathbf{0}_{p / 2}^{\prime}, \mathbf{1}_{p / 2}^{\prime}\right)^{\prime}$ and $\boldsymbol{\omega}_{p}=(1,0,1,0, \ldots, 1,0)_{p \times 1}^{\prime}$, where for any $a$, $\mathbf{1}_{a}=(1, \ldots, 1)_{a \times 1}^{\prime}$ and $\mathbf{0}_{a}=(0, \ldots, 0)_{a \times 1}^{\prime}$. Through appropriate choices of $\mathbf{b}_{p}, \boldsymbol{\delta}_{p}$ and $\omega_{p}$, as applicable, these models can incorporate commonly encountered linear, quadratic and interaction effects.

For each setting, we used two choices of the smoothing method: (a) $\mathcal{T}:=$ $\mathrm{KS}_{2, \mathbf{P}_{2}}$ denoting $\mathrm{KS}$ with 2-dimensional smoothing over $\mathbf{P}_{r}^{\prime} \mathbf{X} \equiv \mathbf{P}_{2}^{\prime} \mathbf{X}$, where $\mathbf{P}_{2}$ was estimated via SIR with $H=100$ slices of equal width, following which $\left\{\widehat{m}_{k}\left(\mathbf{x} ; \widehat{\mathbf{P}}_{r, k}\right)\right\}_{k=1}^{\mathbb{K}}$ were obtained via KS using a Gaussian kernel; (b) $\mathcal{T}:=\mathrm{KM}$ where we let $\mathbf{P}_{r}=I_{p}$ and then estimated $\left\{\widehat{m}_{k}\left(\mathbf{x} ; I_{p}\right)\right\}_{k=1}^{\mathbb{K}}$ using kernel machine (KM) regression based on a radial basis function (RBF) kernel. Throughout, $h$ for $\mathrm{KS}$, and all tuning parameters for $\mathrm{KM}$ were selected via least squares $\mathrm{CV}$. For (a), with $\mathbf{X} \sim \mathcal{N}_{p}\left[\mathbf{0}, I_{p}\right]$, results from Li (1991) imply that the SNP imputation with $r=2$ is sufficient for models (i)-(iii), and insufficient for model (iv). For comparison, we also implemented two other SS estimators: the density ratio based "DRESS" estimator of Kawakita and Kanamori (2013) and the estimator of Sokolovska, Cappé and Yvon (2008) called "MSSL" by Kawakita and Kanamori (2013). The density ratio estimation for the DRESS estimator was implemented using either (i) linear bases $\left\{1,\left(\mathbf{X}_{[j]}\right)_{j=1}^{p}\right\}\left(\right.$ DRESS $\left._{1}\right)$; or (ii) cubic bases $\left\{1,\left(\mathbf{X}_{[j]}^{d}\right)_{j=1, d=1}^{p, 3}\right\}\left(\mathrm{DRESS}_{3}\right)$.

First, we compare the various estimators with respect to their efficiencies based on empirical mean squared error. In Table 1, we present the efficiencies of the proposed SNP and EASE estimators as well as other SS estimators relative to the OLS. As expected, under model mis-specification, our estimators are substantially more efficient than the OLS with the relative efficiency (RE) as high as near 5-fold when $p=10$ and 3-fold when $p=20$, for the nonlinear models. The efficiency gain is generally lower for $p=20$ than for $p=10$, likely a consequence of overfitting of the nonparametric estimators involved in the SNP imputation for larger p. Comparing EASE to SNP, the EASE generally perform better for both linear and nonlinear settings, as expected. Comparing the two smoothers, it appears that $\mathcal{T}:=\mathrm{KM}$ generally attains higher efficiency compared to that of $\mathcal{T}:=\mathrm{KS}_{2, \mathbf{P}_{2}}$. 
TABLE 1

Efficiencies of $\widehat{\boldsymbol{\theta}}_{\left(\mathbf{P}_{r}, \mathbb{K}\right)}(S N P)$ and $\widehat{\boldsymbol{\theta}}_{\left(\mathbf{P}_{r}, \mathbb{K}\right)}^{E}(E A S E)$ using $\mathcal{T}:=\mathrm{KS}_{2, \mathbf{P}_{2}}$ or $\mathcal{T}:=\mathrm{KM}$, as well as DRESS $_{1}$, DRESS $_{3}$ and MSSL, relative to $\widehat{\boldsymbol{\theta}}(O L S)$ with respect to the empirical mean squared error (MSE) under models (i), (ii), (iii) and (iv) each with: (I) $\mathbf{b}_{p}=\mathbf{b}_{p}^{(1)}$ or, (II) $\mathbf{b}_{p}=\mathbf{b}_{p}^{(2)}$

\begin{tabular}{|c|c|c|c|c|c|c|c|c|c|}
\hline \multirow[b]{2}{*}{ Setting } & \multirow[b]{2}{*}{ Models } & \multirow{2}{*}{$\begin{array}{c}\text { OLS } \\
\text { (Ref.) }\end{array}$} & \multicolumn{2}{|c|}{$\left(\mathcal{T}:=\mathbf{K S}_{\mathbf{2}, \mathbf{P}_{\mathbf{2}}}\right)$} & \multicolumn{2}{|c|}{$(\mathcal{T}:=\mathbf{K M})$} & \multicolumn{3}{|c|}{ Other SS estimators } \\
\hline & & & SNP & EASE & SNP & EASE & DRESS $_{1}$ & DRESS $_{3}$ & MSSL \\
\hline \multicolumn{10}{|c|}{ (a) $p=10$} \\
\hline \multirow[t]{4}{*}{ (I) } & Linear & 1 & 0.895 & 0.983 & 0.772 & 0.985 & 0.982 & 0.927 & 0.982 \\
\hline & NL1C & 1 & 4.481 & 4.424 & 4.501 & 5.543 & 1.136 & 1.110 & 1.135 \\
\hline & NL2C & 1 & 2.683 & 2.700 & 4.268 & 5.055 & 1.120 & 1.016 & 1.119 \\
\hline & NL3C & 1 & 2.772 & 2.795 & 4.481 & 5.560 & 1.102 & 1.025 & 1.103 \\
\hline \multirow[t]{4}{*}{ (II) } & Linear & 1 & 0.841 & 0.989 & 0.657 & 0.993 & 0.981 & 0.924 & 0.981 \\
\hline & NL1C & 1 & 4.511 & 4.585 & 4.416 & 5.471 & 1.132 & 1.030 & 1.130 \\
\hline & NL2C & 1 & 3.596 & 3.634 & 4.405 & 5.497 & 1.127 & 1.042 & 1.128 \\
\hline & NL3C & 1 & 3.280 & 3.301 & 4.636 & 5.566 & 1.110 & 1.079 & 1.109 \\
\hline \multicolumn{10}{|c|}{ (b) $p=20$} \\
\hline \multirow[t]{4}{*}{ (I) } & Linear & 1 & 0.673 & 0.986 & 0.740 & 0.981 & 0.956 & 0.866 & 0.956 \\
\hline & NL1C & 1 & 2.256 & 2.288 & 2.680 & 3.630 & 1.035 & 0.920 & 1.035 \\
\hline & NL2C & 1 & 1.414 & 1.388 & 2.661 & 3.544 & 1.032 & 0.922 & 1.033 \\
\hline & NL3C & 1 & 1.539 & 1.531 & 2.605 & 3.510 & 1.049 & 0.931 & 1.051 \\
\hline \multirow[t]{4}{*}{ (II) } & Linear & 1 & 0.519 & 0.991 & 0.609 & 0.989 & 0.958 & 0.872 & 0.958 \\
\hline & NL1C & 1 & 2.290 & 2.346 & 2.669 & 3.660 & 1.032 & 0.908 & 1.031 \\
\hline & NL2C & 1 & 1.899 & 1.917 & 2.766 & 3.963 & 1.036 & 0.917 & 1.036 \\
\hline & NL3C & 1 & 1.937 & 1.949 & 2.682 & 3.702 & 1.046 & 0.958 & 1.046 \\
\hline
\end{tabular}

This is in part due to the high variability in the SIR direction estimation which impacts the performance of the resulting SS estimator in finite samples. Interestingly, none of the existing SS estimators perform well with REs ranging only from about 0.9 to 1.1 across all settings.

We next examine the performance of the proposed inference procedures. In Table 2(a) and (b), we present the bias, empirical standard error (ESE), the average of the estimated standard error (ASE) and the coverage probability (CovP) of the $95 \%$ CIs for each component of $\boldsymbol{\theta}_{0}$ when $p=10$ under the linear and NL2C models. In general, the EASE with both the KS and the KM smoothers have negligible biases although the KM based estimator appears to have slightly lower biases. The ASEs are close to the ESEs and the CovPs are close to the nominal level, suggesting that the variance estimators work well in practice with $\mathbb{K}=5$.

As shown in Table 2(c), the other SS estimators tend to have slightly larger biases and substantially larger standard errors (SEs) compared to our estimators under the NL2C model. 
TABLE 2

Coordinatewise bias, ESE, ASE and CovP of EASE, obtained using $\mathcal{T}:=\mathrm{KS}_{2, \mathbf{P}_{2}}$ or $\mathcal{T}:=\mathrm{KM}$, for estimating $\boldsymbol{\theta}_{0}$ under the linear and NL2C models with $p=10$ and $\mathbf{b}_{p}=\mathbf{b}_{p}^{(1)}$. Shown also are the corresponding bias and ESE of the OLS, as well as the DRESS ${ }_{1}, \mathrm{DRESS}_{3}$ and MSSL estimators

\begin{tabular}{|c|c|c|c|c|c|c|c|c|c|c|}
\hline \multirow[b]{2}{*}{ Parameter } & \multicolumn{2}{|c|}{$\operatorname{OLS}(\widehat{\theta})$} & \multicolumn{4}{|c|}{$\operatorname{EASE}\left(\widehat{\boldsymbol{\theta}}_{\left(\mathbf{P}_{r}, \mathbb{K}\right)}^{E} ; \mathcal{T}:=\mathbf{K S}_{2, \mathbf{P}_{2}}\right)$} & \multicolumn{4}{|c|}{$\operatorname{EASE}\left(\widehat{\theta}_{\left(\mathbf{P}_{r}, \mathbb{K}\right)}^{E} ; \mathcal{T}:=\mathbf{K M}\right)$} \\
\hline & Bias & ESE & Bias & ESE & ASE & CovP & Bias & ESE & ASE & CovP \\
\hline
\end{tabular}

(a) OLS and EASE for the linear model

\begin{tabular}{lrlrlllrlll}
$\alpha_{0}=0$ & -0.001 & 0.043 & -0.001 & 0.043 & 0.044 & 0.95 & 0.000 & 0.043 & 0.044 & 0.96 \\
$\beta_{01}=1$ & 0.002 & 0.044 & -0.003 & 0.045 & 0.044 & 0.94 & 0.004 & 0.047 & 0.044 & 0.93 \\
$\beta_{02}=1$ & -0.001 & 0.044 & -0.005 & 0.044 & 0.044 & 0.94 & 0.000 & 0.045 & 0.044 & 0.95 \\
$\beta_{03}=1$ & -0.001 & 0.046 & -0.005 & 0.046 & 0.044 & 0.95 & -0.004 & 0.045 & 0.044 & 0.94 \\
$\beta_{04}=1$ & -0.002 & 0.045 & -0.006 & 0.045 & 0.044 & 0.94 & 0.001 & 0.047 & 0.044 & 0.94 \\
$\beta_{05}=1$ & -0.004 & 0.048 & -0.008 & 0.049 & 0.044 & 0.92 & -0.001 & 0.046 & 0.044 & 0.95 \\
$\beta_{06}=0$ & -0.000 & 0.045 & -0.001 & 0.045 & 0.044 & 0.94 & 0.001 & 0.045 & 0.044 & 0.95 \\
$\beta_{07}=0$ & 0.003 & 0.046 & 0.003 & 0.046 & 0.044 & 0.93 & 0.001 & 0.043 & 0.044 & 0.96 \\
$\beta_{08}=0$ & -0.001 & 0.045 & -0.001 & 0.045 & 0.044 & 0.95 & -0.000 & 0.048 & 0.044 & 0.94 \\
$\beta_{09}=0$ & -0.002 & 0.047 & -0.002 & 0.048 & 0.044 & 0.94 & 0.000 & 0.045 & 0.045 & 0.95 \\
$\beta_{010}=0$ & 0.003 & 0.045 & 0.003 & 0.045 & 0.044 & 0.94 & -0.002 & 0.045 & 0.045 & 0.94 \\
& & \multicolumn{1}{c}{ (b) OLS and EASE for the NL2C model } & & & \\
$\alpha_{0}=0$ & -0.015 & 0.239 & -0.016 & 0.146 & 0.136 & 0.93 & 0.013 & 0.105 & 0.096 & 0.93 \\
$\beta_{01}=1$ & 0.000 & 0.260 & 0.015 & 0.159 & 0.160 & 0.96 & -0.004 & 0.124 & 0.112 & 0.93 \\
$\beta_{02}=1$ & -0.004 & 0.269 & 0.017 & 0.173 & 0.158 & 0.93 & 0.010 & 0.127 & 0.113 & 0.93 \\
$\beta_{03}=1$ & -0.015 & 0.249 & 0.018 & 0.156 & 0.158 & 0.95 & -0.000 & 0.118 & 0.113 & 0.95 \\
$\beta_{04}=1$ & -0.001 & 0.267 & 0.016 & 0.164 & 0.159 & 0.94 & 0.007 & 0.124 & 0.113 & 0.93 \\
$\beta_{05}=1$ & 0.013 & 0.260 & 0.019 & 0.164 & 0.158 & 0.94 & 0.002 & 0.120 & 0.113 & 0.94 \\
$\beta_{06}=0$ & -0.010 & 0.281 & 0.008 & 0.164 & 0.155 & 0.94 & 0.005 & 0.119 & 0.112 & 0.94 \\
$\beta_{07}=0$ & 0.006 & 0.277 & 0.002 & 0.166 & 0.155 & 0.93 & 0.011 & 0.116 & 0.111 & 0.95 \\
$\beta_{08}=0$ & -0.008 & 0.277 & -0.004 & 0.167 & 0.156 & 0.94 & -0.001 & 0.120 & 0.112 & 0.95 \\
$\beta_{09}=0$ & 0.002 & 0.279 & 0.003 & 0.160 & 0.157 & 0.95 & 0.007 & 0.118 & 0.113 & 0.95 \\
$\beta_{010}=0$ & -0.008 & 0.272 & 0.002 & 0.160 & 0.155 & 0.95 & 0.004 & 0.130 & 0.111 & 0.91
\end{tabular}

\begin{tabular}{|c|c|c|c|c|c|c|c|c|c|c|}
\hline \multicolumn{6}{|c|}{ Linear model } & \multicolumn{5}{|c|}{ NL2C model } \\
\hline DR & & DR & & & & DR & $\mathbf{S S}_{1}$ & DR & & MSSL \\
\hline Bias & ESE & Bias & ESE & Bias & ESE & Bias & ESE & Bias & ESE & Bias \\
\hline
\end{tabular}

(c) All other SS estimators for the models in (a) and (b) above

\begin{tabular}{rrrrrrrrrrrr}
-0.001 & 0.043 & -0.001 & 0.044 & -0.001 & 0.043 & -0.004 & 0.223 & -0.003 & 0.226 & -0.004 & 0.223 \\
-0.002 & 0.044 & -0.001 & 0.046 & -0.002 & 0.044 & -0.014 & 0.266 & -0.009 & 0.279 & -0.014 & 0.266 \\
0.000 & 0.045 & 0.001 & 0.046 & 0.000 & 0.045 & 0.005 & 0.257 & 0.006 & 0.266 & 0.006 & 0.257 \\
0.006 & 0.045 & 0.006 & 0.047 & 0.006 & 0.045 & -0.013 & 0.256 & -0.019 & 0.281 & -0.011 & 0.256 \\
0.003 & 0.045 & 0.003 & 0.046 & 0.003 & 0.045 & -0.005 & 0.262 & -0.007 & 0.274 & -0.005 & 0.262 \\
-0.004 & 0.047 & -0.004 & 0.049 & -0.004 & 0.047 & 0.002 & 0.250 & -0.007 & 0.266 & 0.002 & 0.252 \\
-0.001 & 0.045 & -0.001 & 0.046 & -0.001 & 0.045 & -0.017 & 0.239 & -0.009 & 0.247 & -0.017 & 0.239 \\
-0.000 & 0.048 & -0.001 & 0.050 & -0.000 & 0.048 & -0.022 & 0.260 & -0.019 & 0.270 & -0.022 & 0.260 \\
-0.004 & 0.043 & -0.003 & 0.044 & -0.004 & 0.043 & -0.011 & 0.241 & -0.013 & 0.261 & -0.010 & 0.241 \\
-0.001 & 0.048 & -0.001 & 0.049 & -0.001 & 0.048 & -0.020 & 0.256 & -0.019 & 0.259 & -0.020 & 0.256 \\
-0.003 & 0.047 & -0.003 & 0.049 & -0.003 & 0.047 & -0.020 & 0.252 & -0.022 & 0.269 & -0.020 & 0.252 \\
\hline
\end{tabular}


6.2. Application to EMR data. We applied our proposed SS procedures to an EMR study of rheumatoid arthritis (RA), a systemic auto-immune disease (AD), conducted at the Partners HealthCare [Liao et al. (2010)]. The study cohort consists of 3854 RA patients with blood samples stored. The outcome of interest is the (logarithm of) anti-CCP (antibodies to cyclic citrullinated polypeptide), a biomarker that is often used to determine subtypes of RA. Due to cost constraints, anti-CCP was measured only for a random subset of $n=355$ patients, thereby leading to a SS set-up. To investigate the validity of the MCAR assumption, we report in Table II in the Supplementary Material [Chakrabortty and Cai (2018)] summary measures of the distributions in the labeled and unlabeled data for each of the predictors, as well as p-values from various tests for assessing equality of those distributions. The results suggest that the MCAR assumption is appropriate in this study.

We relate the $\log$ anti-CCP level to a set of $p=24$ clinical variables $\mathbf{X}$ related to ADs, including age, gender, race; total counts of codified and/or narrative mentions extracted from physicians' notes via natural language processing (NLP) for various RA related conditions including RA, Lupus, Polymyalgiarheumatica $(\mathrm{PmR})$, Spondyloarthritis (SpA), as well as various RA medications; indicators of seropositivity and radiological evidence of erosion; mentions of rheumatoid factor $(\mathrm{RF})$, as well as anti-CCP positivity from prior medical history. Since the tests for $\mathrm{RF}$ and anti-CCP were not always ordered, missing indicators for these variables were also included. All count variables were transformed as: $x \rightarrow \log (1+x)$ to increase stability of the model fitting. All predictors were normalized to have unit variance.

We obtained the OLS, the EASE using both $\mathcal{T}:=\mathrm{KS}_{2, \mathbf{P}_{2}}$ and $\mathcal{T}:=\mathrm{KM}$ in the smoothing step, as well as the DRESS 1 estimator for comparison. For EASE, we again used $\mathbb{K}=5$ and for the $\mathrm{KS}_{2, \mathbf{P}_{2}}$ smoother, $\mathbf{P}_{2}$ was obtained using SIR with $H=80$ slices of equal width. In Table 3, we present the coordinatewise estimates of the regression parameters along with their estimated SEs and the corresponding p-values based on these estimates. Overall, the point estimators from all methods are quite close to each other. Our proposed EASE, with both KS and KM smoothers, is substantially more efficient than the OLS across all coordinates with efficiency ranging from about 1.4 to 2.4. The DRESS 1 estimator improved estimation for a few coordinates but the efficiency remains comparable to the OLS for most coordinates. This again suggests the advantage of our proposed estimators compared to both OLS and other SS estimators.

We also estimated the prediction errors (PEs) for each of the fitted linear models based on the aforementioned estimation methods via CV. To remove potential randomness in the $\mathrm{CV}$ partitions, we averaged over 10 replications of leave-5-out $\mathrm{CV}$ estimates. The PE was about 1.28 for EASE with both smoothers, 1.29 for OLS and 1.30 for DRESS 1 . For prediction purposes, we may also directly employ nonparametric estimates of the conditional mean rather than the fitted linear models. The PE in fact is slightly larger when we use $\widehat{m}\left(\mathbf{x} ; \widehat{\mathbf{P}}_{r}\right)$ or $\widehat{\mu}\left(\mathbf{x} ; \widehat{\mathcal{P}}_{r, \mathbb{K}}\right)$. The PE was 1.34 for KS and 1.33 for KM based on $\widehat{m}\left(\mathbf{x} ; \widehat{\mathbf{P}}_{r}\right)$, and 1.30 for KS and 1.28 
TABLE 3

Estimates (Est) of the regression coefficients based on OLS, EASE obtained using either $\mathcal{T}:=\mathrm{KS}_{2, \mathbf{P}_{2}}$ or $\mathcal{T}:=K M$, as well as $\mathrm{DRESS}{ }_{1}$, along with their estimated standard errors (SE) and the corresponding p-values (Pval) for testing the null effect of each predictor. Shown also are the relative efficiencies

(RE) of all the estimators compared to the OLS

\begin{tabular}{|c|c|c|c|c|c|c|c|c|c|c|c|c|c|c|c|}
\hline \multirow[b]{2}{*}{ Predictors } & \multicolumn{3}{|c|}{ OLS } & \multicolumn{3}{|c|}{ EASE $\left(\mathrm{KS}_{2, \mathbf{P}_{2}}\right)$} & \multirow[b]{2}{*}{$\mathbf{R E}$} & \multicolumn{3}{|c|}{ DRESS $_{1}$} & \multirow[b]{2}{*}{$\mathbf{R E}$} & \multicolumn{3}{|c|}{ EASE (KM) } & \multirow[b]{2}{*}{$\mathbf{R E}$} \\
\hline & Est & SE & Pval & Est & SE & Pval & & Est & SE & Pval & & Est & SE & Pval & \\
\hline Age & 0.105 & 076 & 168 & 0.106 & 064 & 099 & 1.40 & 0.094 & 73 & 99 & 1.09 & 0.104 & 0 & 03 & 1.42 \\
\hline Gender & -0.032 & 059 & 589 & -0.028 & 0.050 & 570 & 1.41 & -0.027 & .058 & 638 & 1.04 & -0.031 & & & 1.44 \\
\hline Race & -0.041 & 0.065 & 0.534 & -0.042 & 0.055 & 0.452 & 1.40 & -0.044 & 0.067 & 0.511 & 0.95 & -0.040 & 0.055 & 0.462 & 1.41 \\
\hline Lupus & 0.038 & .066 & 563 & 0.048 & 0.052 & 0.359 & 1.59 & 0.021 & .063 & 0.731 & 1.11 & 0.037 & & 0.464 & 1.70 \\
\hline PmR & -0.075 & 0.044 & 0.088 & -0.076 & 0.031 & 0.013 & 2.07 & -0.074 & 0.031 & 0.016 & 2.10 & -0.075 & 30 & 14 & 2.04 \\
\hline RA & 0.015 & 0.089 & 0.862 & 0.012 & 0.076 & 0.879 & 1.37 & 0.008 & 0.080 & 0.923 & 1.23 & 0.016 & 0.075 & 0.832 & 1.30 \\
\hline $\mathrm{SpA}$ & -0.137 & 0.102 & & -0.133 & 0.072 & & 2.02 & & 0.075 & & 1.82 & & & & 2.37 \\
\hline Other ADs & -0.022 & 0.078 & 0.775 & -0.024 & 0.058 & 0.679 & 1.79 & -0.018 & 0.067 & 0.792 & 1.35 & -0.022 & 0.056 & 0.692 & 1.93 \\
\hline Erosion & 0.076 & 0.070 & 0.278 & 0.078 & 0.059 & 0.184 & 1.44 & 0.085 & 0.069 & 0.221 & 1.03 & 0.076 & & & 1.47 \\
\hline Seropositivity & 0.056 & 0.062 & 0.370 & 0.054 & 0.053 & & 1.37 & 0.041 & 0.061 & 0.496 & 1.05 & 0.055 & 0.052 & & 1.41 \\
\hline Anti- $\mathrm{CCP}_{\text {prior }}$ & 0.572 & 0.136 & 0.000 & 0.557 & 0.110 & 0.000 & 1.54 & 0.567 & 0.123 & 0.000 & 1.23 & 0.568 & 0.107 & 0.000 & 1.60 \\
\hline Anti- $\mathrm{CCP}_{\text {miss }}$ & 0.527 & 0.123 & & 0.520 & & & 1.61 & & & & 1.15 & & & & 1.64 \\
\hline RF & 0.128 & 0.081 & 0.113 & 0.125 & 0.066 & 0.059 & 1.49 & 0.149 & 0.079 & 0.059 & 1.05 & 0.127 & 0.066 & 0.054 & 1.49 \\
\hline $\mathrm{RF}_{\text {miss }}$ & 0.085 & 0.085 & 0.316 & 0.084 & 0.070 & 0.233 & 1.46 & 0.137 & 0.080 & 0.088 & 1.12 & 0.084 & 0.070 & 0.231 & 1.48 \\
\hline Azathioprine & -0.080 & 0.071 & 0.263 & -0.074 & 0.056 & 0.185 & 1.62 & -0.075 & 0.062 & 0.225 & 1.33 & -0.079 & 0.053 & 0.132 & 1.83 \\
\hline Enbrel & 0.138 & 0.070 & 0.048 & 0.133 & 0.058 & 0.021 & 1.48 & 0.136 & 0.073 & 0.064 & 0.91 & 0.137 & 0.057 & 0.017 & 1.49 \\
\hline Gold salts & & 0.050 & & 0.136 & & & 1.37 & & 0.050 & & 1.01 & 0.137 & & & 1.40 \\
\hline Humira & -0.051 & 0.068 & 0.453 & -0.049 & 0.057 & 0.391 & 1.43 & -0.057 & 0.067 & 0.389 & 1.03 & -0.051 & 0.056 & 0.360 & 1.49 \\
\hline Infliximab & 0.003 & 0.069 & 0.968 & 0.008 & 0.057 & 0.887 & 1.50 & 0.000 & 0.067 & 0.994 & 1.07 & 0.003 & 0.055 & 0.959 & 1.57 \\
\hline Leflunomide & -0.027 & 0.069 & 0.697 & -0.023 & 0.058 & 0.693 & 1.40 & -0.031 & 0.071 & 0.660 & 0.93 & -0.026 & 0.057 & 0.644 & 1.45 \\
\hline Methotrexate & -0.021 & 0.073 & 0.775 & -0.024 & 0.061 & 0.699 & 1.42 & -0.025 & 0.073 & 0.728 & 1.01 & -0.022 & 0.060 & 0.720 & 1.46 \\
\hline Plaquenil & -0.043 & 0.069 & 0.540 & -0.038 & 0.057 & 0.503 & 1.47 & -0.044 & 0.070 & 0.532 & 0.98 & -0.042 & 0.057 & 0.460 & 1.48 \\
\hline Sulfasalazine & -0.114 & 0.074 & 0.125 & -0.116 & 0.063 & 0.064 & 1.39 & -0.105 & 0.072 & 0.145 & 1.06 & -0.113 & 0.061 & 0.065 & 1.45 \\
\hline Other meds. & -0.042 & 0.074 & 0.570 & -0.052 & 0.060 & 0.385 & 1.52 & -0.052 & 0.071 & 0.466 & 1.10 & -0.042 & 0.059 & 0.473 & 1.59 \\
\hline
\end{tabular}


for $\mathrm{KM}$ based on $\widehat{\mu}\left(\mathbf{x} ; \widehat{\mathcal{P}}_{r, \mathbb{K}}\right)$. This confirms that while the linear model may be mis-specified, it may often be preferable to nonparametric models in practice as it may achieve simplicity without substantial loss in prediction performance.

7. Discussion. We have developed in this paper an efficient and adaptive estimation strategy for the SS linear regression problem. The adaptive property possessed by the proposed EASE is crucial for advocating "safe" use of the unlabeled data and is often unaddressed in the existing literature. In general, the magnitude of the efficiency gain with EASE depends on the inherent degree of nonlinearity in $\mathbb{E}(Y \mid \mathbf{X})$ and the extent of sufficiency of the underlying SNP imputation. In particular, if the imputation is sufficient or the working linear model is correct, $\widehat{\boldsymbol{\theta}}_{\left(\mathbf{P}_{r}, \mathbb{K}\right)}^{E}$ is further optimal among a wide class of estimators. We obtained theoretical results along with IF expansions for $\widehat{\boldsymbol{\theta}}_{\left(\mathbf{P}_{r}, \mathbb{K}\right)}$ and $\widehat{\boldsymbol{\theta}}_{\left(\mathbf{P}_{r}, \mathbb{K}\right)}^{E}$ substantiating all our claims and also validated them based on numerical studies. The double CV method further facilitates accurate inference, overcoming potential over-fitting issues in finite samples due to smoothing. An R code for implementing EASE is available upon request.

The proposed SNP imputation, the key component of EASE, apart from being flexible and scalable, enjoys several useful properties. The refitting step and CV play a crucial role in reducing the bias of $\widehat{\boldsymbol{\theta}}_{\left(\mathbf{P}_{r}, \mathbb{K}\right)}$, and for $\mathcal{T}:=\mathrm{KS}$ in particular, eradicate any under-smoothing or higher order kernel requirements: two undesirable, yet often inevitable, conditions required for $n^{\frac{1}{2}}$-consistency of two-step estimators based on a first step of smoothing. Theorem 4.2, apart from showing the distinct behaviour of $\widehat{\boldsymbol{\theta}}_{\left(\mathbf{P}_{r}, 1\right)}$ compared to $\widehat{\boldsymbol{\theta}}_{\left(\mathbf{P}_{r}, \mathbb{K}\right)}$ for $\mathbb{K} \geq 2$, also highlights the key role of $\mathrm{CV}$ in completely removing kernel order restrictions, apart from addressing over-fitting issues. The error rates in the results of Theorems 4.1-4.2 are quite sharp and account for any estimation error from $\widehat{\mathbf{P}}_{r}$. The regularity conditions required are also fairly mild and standard in the literature. The continuity assumption on $\mathbf{X}$ in Sections 3.1 and 4 is mostly for the convenience of proofs, and the results continue to hold for more general $\mathbf{X}$. Lastly, while we have focussed here on linear regression for simplicity, our methods can indeed be easily adapted to other regression problems such as logistic regression for binary outcomes.

When the goal is solely that of prediction, one obviously does not have to employ linear regression models, and models that incorporate nonlinear effects can be helpful. For such settings, the estimators $\widehat{m}\left(\mathbf{x} ; \widehat{\mathbf{P}}_{r}\right)$ or $\widehat{\mu}\left(\mathbf{x} ; \widehat{\mathcal{P}}_{r, \mathbb{K}}\right)$, obtained as by-products of our SNP imputation, can themselves serve as potentially useful nonlinear predictors. These SNP estimators may substantially outperform naive nonparametric estimators such as a $p$-dimensional KS estimator, as demonstrated in Table III of the Supplementary Material [Chakrabortty and Cai (2018)] for the models considered in our simulation studies. In practice, when the covariates are substantially correlated and the dimension of $p$ is not small as in the EMR example, it is unclear whether nonlinear models necessarily provide better prediction 
performance than the linear models. Under such settings, the linear model also has a clear advantage due to its simplicity. Furthermore, while prediction is a vitally important goal of predictive modeling, association analysis under interpretable models is key to clinical studies for discovery research and efficient estimation of the corresponding model parameters remains an important task.

We end with a comment on the choice of $\mathbb{K} \geq 2$ in $\widehat{\boldsymbol{\theta}}_{\left(\mathbf{P}_{r}, \mathbb{K}\right)}$. While (3.11) holds for any $\mathbb{K} \geq 2$, the error term in (3.11) depends on $\mathbb{K}$ through $c_{n_{\mathbb{K}}^{-}}$and more precisely, through $\widetilde{c}_{n_{\mathbb{K}}}=\mathbb{K}^{\frac{1}{2}} c_{n_{\mathbb{K}}^{-}}$. Since $\mathbb{K}$ is fixed, $c_{n_{\mathbb{K}}}$ and $\widetilde{c}_{n_{\mathbb{K}}}$ are asymptotically equivalent. But for a given $n, c_{n_{\mathbb{K}}^{-}}$is expected to decrease with $\mathbb{K}$, while $\widetilde{c}_{n_{\mathbb{K}}}$ is likely to increase. It is however desirable that both are small since $c_{n_{\mathbb{K}}}$ inherently controls the efficiency of the SNP imputation, while $\widetilde{c}_{n_{\mathbb{K}}^{-}}$directly controls the bias of $\widehat{\boldsymbol{\theta}}_{\left(\mathbf{P}_{r}, \mathbb{K}\right)}$. Hence, a reasonable choice of $\mathbb{K} \geq 2$ may be based on minimizing: $\left(c_{n_{\mathbb{K}}^{-}}^{2}+\lambda \widetilde{c}_{n_{\mathbb{K}}^{-}}^{2}\right)$ for some $\lambda \geq 0$. Since the (first-order) asymptotic variance of $\widehat{\boldsymbol{\theta}}_{\left(\mathbf{P}_{r}, \mathbb{K}\right)}$ is independent of $\mathbb{K}$, this is equivalent to a penalized minimization of the asymptotic MSE of $\widehat{\boldsymbol{\theta}}_{\left(\mathbf{P}_{r}, \mathbb{K}\right)}$ with $\lambda$ denoting the weightage of the (lower order) bias relative to the (first-order) variance. In general, the optimal $\mathbb{K}$ should be inversely related to $\lambda$. Conversely, choice of any $\mathbb{K}$ may be viewed to have an associated regularization effect (through $\lambda$ ) resulting in a "variance-bias trade-off" with smaller $\mathbb{K}$ leading to lower bias at the cost of some efficiency, and higher $\mathbb{K}$ leading to improved efficiency in lieu of some bias. In practice, we find that $\mathbb{K}=5$ works well, and $\mathbb{K}=10$ tends to give slightly smaller MSE at the cost of increased bias.

\section{APPENDIX}

A.1. Preliminaries. The following Lemmas A.1-A.3 would be useful in the proofs of the main theorems. The proofs of these lemmas, as well as Theorems 3.1, 4.1 and 4.2, can be found in the Supplementary Material [Chakrabortty and Cai (2018)].

LEMMA A.1. Let $\mathbf{Z} \in \mathbb{R}^{l}$ be any random vector and $\mathbf{g}(\mathbf{Z}) \in \mathbb{R}^{d}$ be any measurable function of $\mathbf{Z}$, where $l$ and $d$ are fixed. Let $\mathbb{S}_{n}=\left\{\mathbf{Z}_{i}\right\}_{i=1}^{n} \Perp \mathbb{S}_{m}=\left\{\mathbf{Z}_{j}\right\}_{j=1}^{m}$ be two random samples of $n$ and $m$ i.i.d. observations of $\mathbf{Z}$, respectively. Let $\widehat{\mathbf{g}}_{n}(\cdot)$ be any estimator of $\mathbf{g}(\cdot)$ based on $\mathbb{S}_{n}$ such that the random sequence: $\widehat{T}_{n} \equiv \sup _{\mathbf{z} \in \chi}\left\|\widehat{\mathbf{g}}_{n}(\mathbf{z})\right\|$ is $O_{p}(1)$, where $\chi \subseteq \mathbb{R}^{l}$ denotes the support of $\mathbf{Z}$. Let $\widehat{\mathbf{G}}_{n, m}$ denote the (double) random sequence: $m^{-1} \sum_{\mathbf{Z}_{j} \in \mathbb{S}_{m}} \widehat{\mathbf{g}}_{n}\left(\mathbf{Z}_{j}\right)$, and let $\overline{\mathbf{G}}_{n}$ denote the random sequence: $\mathbb{E}_{\mathbb{S}_{m}}\left(\widehat{\mathbf{G}}_{n, m}\right)=\mathbb{E}_{\mathbf{Z}}\left\{\widehat{\mathbf{g}}_{n}(\mathbf{Z})\right\}$, where $\mathbb{E}_{\mathbf{Z}}(\cdot)$ denotes expectation w.r.t. $\mathbf{Z} \in \mathbb{S}_{m} \Perp \mathbb{S}_{n}$, and all expectations involved are assumed to be finite almost surely (a.s.) $\left[\mathbb{S}_{n}\right] \forall n$.

Then: (a) $\mathbf{G}_{n, m}-\overline{\mathbf{G}}_{n}=O_{p}\left(m^{-\frac{1}{2}}\right)$, and (b) as long as $g(\cdot)$ has finite 2 nd moments, $m^{-1} \sum_{\mathbf{Z}_{j} \in \mathbb{S}_{m}} \mathbf{g}\left(\mathbf{Z}_{j}\right)-\mathbb{E}_{\mathbf{Z}}\{\mathbf{g}(\mathbf{Z})\}=O_{p}\left(m^{-\frac{1}{2}}\right)$. 
Controlling empirical processes indexed by KS estimators. The next two lemmas would be useful in the proof of Theorem 4.2. They may also be of more general use in other applications that involve controlling empirical processes indexed by kernel estimators - both linear and ratio-type estimators, where the smoothing is further allowed to be performed over a possibly lower dimensional and estimated transformation of the original covariate $\mathbf{X}$. These allowances make the technical analyses of such processes considerably more involved and nuanced. The results of these lemmas and the techniques used in their proofs may therefore be of independent general interest.

Suppose Assumption 2.1(a) holds, and consider the KS framework introduced in Section 4. Let $\widetilde{\varphi}_{\mathbf{P}_{r}}^{(\varrho)}(\mathbf{w})=\left(n h^{r}\right)^{-1} \sum_{i=1}^{n} K_{h}\left(\mathbf{w}, \mathbf{P}_{r}^{\prime} \mathbf{X}_{i}\right) Y_{i}^{\varrho}$, for $\varrho=0,1$. Let $\tilde{f}_{\mathbf{P}_{r}}(\cdot)=\widetilde{\varphi}_{\mathbf{P}_{r}}^{(0)}(\cdot), \tilde{l}_{\mathbf{P}_{r}}(\cdot)=\widetilde{\varphi}_{\mathbf{P}_{r}}^{(1)}(\cdot)$ and $\tilde{m}_{\mathbf{P}_{r}}(\cdot)=\tilde{l}_{\mathbf{P}_{r}}(\cdot) / \tilde{f}_{\mathbf{P}_{r}}(\cdot)$. Next, let $\varphi_{\mathbf{P}_{r}}^{(0)}(\cdot)=$ $f_{\mathbf{P}_{r}}(\cdot)$ and $\varphi_{\mathbf{P}_{r}}^{(1)}(\cdot)=l_{\mathbf{P}_{r}}(\cdot)$, where $l_{\mathbf{P}_{r}}(\cdot)=m_{\mathbf{P}_{r}}(\cdot) f_{\mathbf{P}_{r}}(\cdot)$. For each $\varrho \in\{0,1\}$, let $\varphi^{(\varrho)}\left(\mathbf{x} ; \mathbf{P}_{r}\right)=\varphi_{\mathbf{P}_{r}}^{(\varrho)}\left(\mathbf{P}_{r}^{\prime} \mathbf{x}\right)$ and $\widetilde{\varphi}^{(\varrho)}\left(\mathbf{x} ; \mathbf{P}_{r}\right)=\widetilde{\varphi}_{\mathbf{P}_{r}}^{(\varrho)}\left(\mathbf{P}_{r}^{\prime} \mathbf{x}\right)$. Further, let $\tilde{f}(\cdot)=\widetilde{\varphi}^{(0)}(\cdot)$, $\widetilde{l}(\cdot)=\widetilde{\varphi}^{(1)}(\cdot)$ and $\tilde{m}(\cdot)=\tilde{l}(\cdot) / \tilde{f}(\cdot)$.

Lastly, let $\mathbb{P}_{n}$ denote the empirical probability measure on $\mathbb{R}^{p}$ based on $\left\{\mathbf{X}_{i}\right\}_{i=1}^{n}$, and for any measurable (and possibly vector-valued) function $\boldsymbol{\gamma}(\cdot)$ of $\mathbf{X}$, where $\boldsymbol{\gamma}(\cdot)$ can be random itself, let $\mathbb{G}_{n}^{*}(\boldsymbol{\gamma})=n^{\frac{1}{2}} \int \boldsymbol{\gamma}(\mathbf{x})\left(\mathbb{P}_{n}-\mathbb{P}_{\mathbf{X}}\right)(d \mathbf{x})$, the (centered) $n^{\frac{1}{2}}$ scaled empirical process indexed by $\boldsymbol{\gamma}(\cdot)$. Lemmas A.2-A.3 together, among other more general implications, establish explicit rates of convergence of the quantity $\mathbb{G}_{n}^{*}\{\widehat{g}(\cdot)-g(\cdot)\}$, for any linear or ratio-type kernel estimator $\widehat{g}(\cdot)$ of the type discussed above and its corresponding target $g(\cdot)$.

LEMMA A.2. Consider the set-up introduced above. For any fixed integer $d \geq$ 1 , let $\lambda(\cdot)$ be any $\mathbb{R}^{d}$-valued measurable function of $\mathbf{X}$ that is bounded a.s. $\left[\mathbb{P}_{\mathbf{X}}\right]$. Define $b_{n}^{(1)}=n^{-\frac{1}{2}} h^{-r}+h^{q}$ and $a_{n, 2}=(\log n)^{\frac{1}{2}}\left(n h^{r}\right)^{-\frac{1}{2}}+h^{q}$. Assume $b_{n}^{(1)}=o(1)$ for (A.1) and $n^{\frac{1}{2}} a_{n, 2}^{2}=o(1)$ for (A.2) below. Then, under Assumption 4.1(i)-(v), and $\forall \varrho \in\{0,1\}$,

$$
\begin{gathered}
\mathbb{G}_{n}^{*}\left[\lambda(\cdot)\left\{\widetilde{\varphi}^{(\varrho)}\left(\cdot ; \mathbf{P}_{r}\right)-\varphi^{(\varrho)}\left(\cdot ; \mathbf{P}_{r}\right)\right\}\right]=O_{p}\left(b_{n}^{(1)}\right)=o_{p}(1) \quad \text { and } \\
\mathbb{G}_{n}^{*}\left[\lambda(\cdot)\left\{\widetilde{m}\left(\cdot ; \mathbf{P}_{r}\right)-m\left(\cdot ; \mathbf{P}_{r}\right)\right\}\right]=O_{p}\left(n^{\frac{1}{2}} a_{n, 2}^{2}\right)=o_{p}(1) .
\end{gathered}
$$

Let $\widehat{\varphi}^{(\varrho)}\left(\mathbf{x} ; \widehat{\mathbf{P}}_{r}\right)=\left(n h^{r}\right)^{-1} \sum_{i=1}^{n} K_{h}\left(\widehat{\mathbf{P}}_{r}^{\prime} \mathbf{x}, \widehat{\mathbf{P}}_{r}^{\prime} \mathbf{X}_{i}\right) Y_{i}^{\varrho} \forall \varrho \in\{0,1\}$, where $\widehat{\mathbf{P}}_{r}$ is as in Section 3.2 and all other notation are the same as in the set up of Lemma A.2. Let $\widehat{f}\left(\mathbf{x} ; \widehat{\mathbf{P}}_{r}\right)=\widehat{\varphi}^{(0)}\left(\mathbf{x} ; \widehat{\mathbf{P}}_{r}\right)$ and $\widehat{l}\left(\mathbf{x} ; \widehat{\mathbf{P}}_{r}\right)=\widehat{\varphi}^{(1)}\left(\mathbf{x} ; \widehat{\mathbf{P}}_{r}\right)$. Then we have the following.

LEMMA A.3. Consider the set-up of Lemma A.2. Let $\widehat{\varphi}^{(\varrho)}\left(\mathbf{x} ; \widehat{\mathbf{P}}_{r}\right)$ be as above, and let $\lambda(\cdot)$ be as in Lemma A.2. Suppose $\left(\widehat{\mathbf{P}}_{r}-\mathbf{P}_{r}\right)=O_{p}\left(\alpha_{n}\right)$ for some $\alpha_{n}=o(1)$. Assume $b_{n}^{(2)}=o(1)$, where $b_{n}^{(2)}=\alpha_{n}+n^{-\frac{1}{2}} \alpha_{n} h^{-(r+1)}+n^{\frac{1}{2}} \alpha_{n}^{2}\left(h^{-2}+n^{-1} h^{-(r+2)}\right)$. Then, under Assumption 4.1,

$$
\mathbb{G}_{n}^{*}\left[\lambda(\cdot)\left\{\widehat{\varphi}^{(\varrho)}\left(\cdot ; \widehat{\mathbf{P}}_{r}\right)-\widetilde{\varphi}^{(\varrho)}\left(\cdot ; \mathbf{P}_{r}\right)\right\}\right]=O_{p}\left(b_{n}^{(2)}\right)=o_{p}(1) \quad \forall \varrho \in\{0,1\} .
$$


A.2. Proof of Theorem 3.2. Let $\boldsymbol{\Gamma}_{n}=\frac{1}{n} \sum_{i=1}^{n} \overrightarrow{\mathbf{X}}_{i} \overrightarrow{\mathbf{X}}_{i}^{\prime}$, and

$$
\begin{aligned}
\mathbf{T}_{n}^{(1)} & =\frac{1}{n} \sum_{i=1}^{n} \overrightarrow{\mathbf{X}}_{i}\left\{Y_{i}-\mu\left(\mathbf{X}_{i} ; \mathbf{P}_{r}\right)\right\}, \\
\mathbf{T}_{n, \mathbb{K}}^{(2)} & =\frac{1}{n} \sum_{k=1}^{\mathbb{K}} \sum_{i \in \mathcal{I}_{k}} \overrightarrow{\mathbf{X}}_{i} \widehat{\Delta}_{k}\left(\mathbf{X}_{i} ; \mathbf{P}_{r}, \widehat{\mathbf{P}}_{r, k}\right) .
\end{aligned}
$$

Then, using (3.4)-(3.8), it is straightforward to see that:

(A.4) $\mathbb{E}\left[\overrightarrow{\mathbf{X}}\left\{Y-\mu\left(\mathbf{X} ; \mathbf{P}_{r}\right)\right\}\right] \equiv \mathbb{E}\left[\overrightarrow{\mathbf{X}}\left\{Y-m\left(\mathbf{X} ; \mathbf{P}_{r}\right)-\overrightarrow{\mathbf{X}}^{\prime} \boldsymbol{\eta}_{\mathbf{P}_{r}}\right\}\right]=\mathbf{0}$ and

$$
\boldsymbol{\Gamma}_{n}\left(\widehat{\boldsymbol{\eta}}_{\left(\mathbf{P}_{r}, \mathbb{K}\right)}-\eta_{\mathbf{P}_{r}}\right)=\mathbf{T}_{n}^{(1)}-\mathbf{T}_{n, \mathbb{K}}^{(2)} .
$$

Under (A.4), Assumptions 2.1(a) and (i), it follows from Lemma A.1(b) that $\mathbf{T}_{n}^{(1)}=$ $O_{p}\left(n^{-\frac{1}{2}}\right)$. Next, due to assumption (ii) and boundedness of $\mathbf{X}$,

$$
\left\|\mathbf{T}_{n, \mathbb{K}}^{(2)}\right\| \leq n^{-1} \sum_{k=1}^{\mathbb{K}} \sum_{i \in \mathcal{I}_{k}} \sup _{\mathbf{x} \in \mathcal{X}}\left\{\|\overrightarrow{\mathbf{x}}\|\left|\widehat{\Delta}_{k}\left(\mathbf{x} ; \mathbf{P}_{r}, \widehat{\mathbf{P}}_{r, k}\right)\right|\right\}=O_{p}\left(c_{n_{\mathbb{K}}^{-}}\right) .
$$

Finally, under Assumption 2.1(a), we have $\boldsymbol{\Gamma}_{n}=\boldsymbol{\Gamma}+O_{p}\left(n^{-\frac{1}{2}}\right)$ using Lemma A.1(b). Further, since $\boldsymbol{\Gamma}_{n}$ is invertible a.s., $\boldsymbol{\Gamma}_{n}^{-1}=\boldsymbol{\Gamma}^{-1}+O_{p}\left(n^{-\frac{1}{2}}\right)$. Using all these facts, we then have $\left(\widehat{\boldsymbol{\eta}}_{\left(\mathbf{P}_{r}, \mathbb{K}\right)}-\eta_{\mathbf{P}_{r}}\right)=\boldsymbol{\Gamma}_{n}^{-1}\left(\mathbf{T}_{n}^{(1)}-\mathbf{T}_{n, \mathbb{K}}^{(2)}\right)=\boldsymbol{\Gamma}^{-1}\left(\mathbf{T}_{n}^{(1)}-\right.$ $\left.\mathbf{T}_{n, \mathbb{K}}^{(2)}\right)+O_{p}\left\{n^{-\frac{1}{2}}\left(n^{-\frac{1}{2}}+c_{n_{\mathbb{K}}^{-}}\right)\right\}$. Thus,

$$
\left(\widehat{\boldsymbol{\eta}}_{\left(\mathbf{P}_{r}, \mathbb{K}\right)}-\eta_{\mathbf{P}_{r}}\right)=\boldsymbol{\Gamma}^{-1}\left(\mathbf{T}_{n}^{(1)}-\mathbf{T}_{n, \mathbb{K}}^{(2)}\right)+O_{p}\left(n^{-1}+n^{-\frac{1}{2}} c_{n_{\mathbb{K}}^{-}}\right) .
$$

Next, let us define

$$
\begin{aligned}
\boldsymbol{\Gamma}_{N} & =N^{-1} \sum_{j=n+1}^{n+N} \overrightarrow{\mathbf{X}}_{j} \overrightarrow{\mathbf{X}}_{j}^{\prime}, \\
\mathbf{R}_{N}^{(1)} & =N^{-1} \sum_{j=n+1}^{n+N} \overrightarrow{\mathbf{X}}_{j}\left\{\mu\left(\mathbf{X}_{j} ; \mathbf{P}_{r}\right)-\overrightarrow{\mathbf{X}}_{j}^{\prime} \boldsymbol{\theta}_{0}\right\} \quad \text { and } \\
\widehat{\mathbf{R}}_{N, n}^{(\mathbb{K})} & =N^{-1} \sum_{j=n+1}^{n+N} \overrightarrow{\mathbf{X}}_{j}\left\{\widehat{\mu}\left(\mathbf{X}_{j} ; \widehat{\mathcal{P}}_{r, \mathbb{K}}\right)-\mu\left(\mathbf{X}_{j} ; \mathbf{P}_{r}\right)\right\} .
\end{aligned}
$$

Then, using (3.7), we have

$$
\boldsymbol{\Gamma}_{N}\left(\widehat{\boldsymbol{\theta}}_{\left(\mathbf{P}_{r}, \mathbb{K}\right)}-\boldsymbol{\theta}_{0}\right)=N^{-1} \sum_{j=n+1}^{n+N} \overrightarrow{\mathbf{X}}_{j}\left[\widehat{\mu}\left(\mathbf{X}_{j} ; \widehat{\mathcal{P}}_{r, \mathbb{K}}\right)-\overrightarrow{\mathbf{X}}_{j}^{\prime} \boldsymbol{\theta}_{0}\right]=\mathbf{R}_{N}^{(1)}+\widehat{\mathbf{R}}_{N, n}^{(\mathbb{K})}
$$


Next, using (3.4)-(3.8), we have $\widehat{\mathbf{R}}_{N, n}^{(\mathbb{K})}=\boldsymbol{\Gamma}_{N}\left(\widehat{\boldsymbol{\eta}}_{\left(\mathbf{P}_{r}, \mathbb{K}\right)}-\boldsymbol{\eta}_{\mathbf{P}_{r}}\right)+\widehat{\mathbf{S}}_{N, n}^{(\mathbb{K})}$, where

$$
\widehat{\mathbf{S}}_{N, n}^{(\mathbb{K})}=\mathbb{K}^{-1} \sum_{k=1}^{\mathbb{K}}\left\{N^{-1} \sum_{j=n+1}^{n+N} \overrightarrow{\mathbf{X}}_{j} \widehat{\Delta}_{k}\left(\mathbf{X}_{j} ; \mathbf{P}_{r}, \widehat{\mathbf{P}}_{r, k}\right)\right\}
$$

Hence, we have: $\boldsymbol{\Gamma}_{N}\left(\widehat{\boldsymbol{\theta}}_{\left(\mathbf{P}_{r}, \mathbb{K}\right)}-\boldsymbol{\theta}_{0}\right)=\boldsymbol{\Gamma}_{N}\left(\widehat{\boldsymbol{\eta}}_{\left(\mathbf{P}_{r}, \mathbb{K}\right)}-\boldsymbol{\eta}_{\mathbf{P}_{r}}\right)+\mathbf{R}_{N}^{(1)}+\widehat{\mathbf{S}}_{N, n}^{(\mathbb{K})}$.

Now, under Assumptions (i)-(ii) and Assumption 2.1(a), we have

$$
\text { (I) } \sum_{k=1}^{\mathbb{K}} \sup _{\mathbf{x} \in \mathcal{X}}\left\|\overrightarrow{\mathbf{x}} \widehat{\Delta}_{k}\left(\mathbf{x} ; \mathbf{P}_{r}, \widehat{\mathbf{P}}_{r, k}\right)\right\|=O_{p}(1),
$$

so that using Lemma A.1(a), $\widehat{\mathbf{S}}_{N, n}^{(\mathbb{K})}=\mathbb{K}^{-1} \sum_{k=1}^{\mathbb{K}} \widehat{\mathbf{S}}_{n, k}^{*}+O_{p}\left(N^{-\frac{1}{2}}\right)$, where $\widehat{\mathbf{S}}_{n, k}^{*}=$ $\mathbb{E}_{\mathbf{X}}\left\{\overrightarrow{\mathbf{X}} \widehat{\Delta}_{k}\left(\mathbf{X} ; \mathbf{P}_{r}, \widehat{\mathbf{P}}_{r, k}\right)\right\} \forall 1 \leq k \leq \mathbb{K} ;$

$$
\text { (II) } \mathbf{R}_{N}^{(1)}=\mathbb{E}\left[\overrightarrow{\mathbf{X}}\left\{\mu\left(\mathbf{X} ; \mathbf{P}_{r}\right)-\overrightarrow{\mathbf{X}}^{\prime} \boldsymbol{\theta}_{0}\right\}\right]+O_{p}\left(N^{-\frac{1}{2}}\right)=O_{p}\left(N^{-\frac{1}{2}}\right)
$$

from Lemma A.1(b) and $\mathbb{E}\left[\overrightarrow{\mathbf{X}}\left\{\mu\left(\mathbf{X} ; \mathbf{P}_{r}\right)-\overrightarrow{\mathbf{X}}^{\prime} \boldsymbol{\theta}_{0}\right\}\right]=\mathbf{0}$ due to (A.4) and Definition 2.1; and lastly, (III) $\boldsymbol{\Gamma}_{N}^{-1}=\boldsymbol{\Gamma}^{-1}+O_{p}\left(N^{-\frac{1}{2}}\right)$. It then follows from (I)-(III) that

$$
\widehat{\boldsymbol{\theta}}_{\left(\mathbf{P}_{r}, \mathbb{K}\right)}-\boldsymbol{\theta}_{0}=\left(\widehat{\boldsymbol{\eta}}_{\left(\mathbf{P}_{r}, \mathbb{K}\right)}-\boldsymbol{\eta}_{\mathbf{P}_{r}}\right)+\mathbb{K}^{-1} \boldsymbol{\Gamma}^{-1} \sum_{k=1}^{\mathbb{K}} \widehat{\mathbf{S}}_{n, k}^{*}+O_{p}\left(N^{-\frac{1}{2}}\right)
$$

Using (A.6) and (3.9) in (A.7), we then have

$$
\begin{aligned}
\widehat{\boldsymbol{\theta}}_{\left(\mathbf{P}_{r}, \mathbb{K}\right)}-\boldsymbol{\theta}_{0} & \\
= & \frac{1}{n} \sum_{i=1}^{n} \boldsymbol{\psi}\left(\mathbf{Z}_{i} ; \mathbf{P}_{r}\right)-\boldsymbol{\Gamma}^{-1} \frac{1}{\mathbb{K}} \sum_{k=1}^{\mathbb{K}}\left\{\frac{1}{n_{\mathbb{K}}} \sum_{i \in \mathcal{I}_{k}} \widehat{\mathbf{G}}_{k}\left(\mathbf{X}_{i}\right)\right\}+O_{p}\left(b_{n}, \mathbb{K}\right),
\end{aligned}
$$

where $b_{n, \mathbb{K}}=n^{-1}+n^{-\frac{1}{2}} c_{n_{\mathbb{K}}^{-}}+N^{-\frac{1}{2}}$. It follows, as claimed in (3.10), that

$$
n^{\frac{1}{2}}\left(\widehat{\boldsymbol{\theta}}_{\left(\mathbf{P}_{r}, \mathbb{K}\right)}-\boldsymbol{\theta}_{0}\right)=n^{-\frac{1}{2}} \sum_{i=1}^{n} \boldsymbol{\psi}\left(\mathbf{Z}_{i} ; \mathbf{P}_{r}\right)-\boldsymbol{\Gamma}^{-1} \mathbb{G}_{n, \mathbb{K}}+O_{p}\left(c_{n, \mathbb{K}}^{*}\right) .
$$

We next show that $\mathbb{G}_{n, \mathbb{K}}=O_{p}\left(c_{n_{\mathbb{K}}}\right)$ for any fixed $\mathbb{K} \geq 2$. To this end, let $\mathbb{T}_{k}^{(n)}=$ $\left(n_{\mathbb{K}}\right)^{-\frac{1}{2}} \sum_{i \in \mathcal{I}_{k}} \widehat{\mathbf{G}}_{k}\left(\mathbf{X}_{i}\right), \widehat{D}_{k}=\sup _{\mathbf{x} \in \mathcal{X}}\left|\widehat{\Delta}_{k}\left(\mathbf{x} ; \mathbf{P}_{r}, \widehat{\mathbf{P}}_{r, k}\right)\right|$ and $C=\sup _{\mathbf{x} \in \mathcal{X}}\|\overrightarrow{\mathbf{x}}\|<$ $\infty$. For any subset $\mathcal{A} \subseteq \mathcal{L}$, let $\mathbb{P}_{\mathcal{A}}$ denote the joint distribution of the observations in $\mathcal{A}$, and let $\mathbb{E}_{\mathcal{A}}(\cdot)$ denote expectation w.r.t. $\mathbb{P}_{\mathcal{A}}$. By definition, $\mathbb{G}_{n, \mathbb{K}}=$ $\mathbb{K}^{-\frac{1}{2}} \sum_{k=1}^{\mathbb{K}} \mathbb{T}_{k}^{(n)}=O_{p}\left(c_{n_{\mathbb{K}}^{-}}\right)$if and only if given any $\varepsilon>0, \exists M_{\varepsilon}>0$ such that 
$\mathbb{P}\left(\left\|\mathbb{G}_{n, \mathbb{K}}\right\|>M_{\varepsilon} c_{n_{\mathbb{K}}^{-}}\right) \leq \varepsilon \forall n$. Note that for any $M>0$,

(A.9)

$$
\mathbb{P}\left(\left\|\mathbb{G}_{n, \mathbb{K}}\right\|>M c_{n_{\mathbb{K}}^{-}}\right)
$$

$$
\begin{aligned}
& \leq \mathbb{P}\left(\mathbb{K}^{-\frac{1}{2}} \sum_{k=1}^{\mathbb{K}}\left\|\mathbb{T}_{k}^{(n)}\right\|>M c_{n_{\mathbb{K}}^{-}}\right) \\
& \leq \sum_{k=1}^{\mathbb{K}} \mathbb{P}\left(\mathbb{K}^{-\frac{1}{2}}\left\|\mathbb{T}_{k}^{(n)}\right\|>\frac{M c_{n_{\mathbb{K}}^{-}}}{\mathbb{K}}\right) \leq \sum_{k=1}^{\mathbb{K}} \sum_{l=1}^{p+1} \mathbb{P}\left\{\left|\mathbb{T}_{k[l]}^{(n)}\right|>\frac{M c_{n_{\mathbb{K}}}}{\mathbb{K}^{\frac{1}{2}}(p+1)^{\frac{1}{2}}}\right\} \\
& =\sum_{k=1}^{\mathbb{K}} \sum_{l=1}^{p+1} \mathbb{E}_{\mathcal{L}_{k}^{-}}\left[\mathbb{P}_{\mathcal{L}_{k}}\left\{\left|\mathbb{T}_{k[l]}^{(n)}\right|>\frac{M c_{n_{\mathbb{K}}^{-}}}{\mathbb{K}^{\frac{1}{2}}(p+1)^{\frac{1}{2}}} \mid \mathcal{L}_{k}^{-}\right\}\right],
\end{aligned}
$$

where the steps follow from repeated use of Bonferroni's inequality and other standard arguments. Now, conditional on $\mathcal{L}_{k}^{-}\left(\Perp \mathcal{L}_{k}\right.$, with $\left.\mathbb{K} \geq 2\right), n_{\mathbb{K}}^{\frac{1}{2}} \mathbb{T}_{k}^{(n)}$ is a centered sum of the i.i.d. random vectors $\left\{\overrightarrow{\mathbf{X}}_{i} \widehat{\Delta}_{k}\left(\mathbf{X}_{i} ; \mathbf{P}_{r}, \widehat{\mathbf{P}}_{r, k}\right)\right\}_{i \in \mathcal{I}_{k}}$ which, due to assumption (ii) and the compactness of $\mathcal{X}$, are bounded by: $C \widehat{D}_{k}<\infty$ a.s. $\left[\mathbb{P}_{\mathcal{L}_{k}^{-}}\right] \forall k, n$. Hence, applying Hoeffding's inequality to $\mathbb{T}_{k[l]}^{(n)} \forall l$, we have

$$
\begin{array}{r}
\mathbb{P}_{\mathcal{L}_{k}}\left\{\left|\mathbb{T}_{k[l]}^{(n)}\right|>\frac{M c_{n_{\mathbb{K}}}}{\mathbb{K}^{\frac{1}{2}}(p+1)^{\frac{1}{2}}} \mid \mathcal{L}_{k}^{-}\right\} \\
\leq 2 \exp \left\{-\frac{M^{2} c_{n_{\bar{K}}^{2}}^{2}}{2(p+1) \mathbb{K}^{2} \widehat{D}_{k}^{2}}\right\}
\end{array}
$$

$$
\text { a.s. }\left[\mathbb{P}_{\mathcal{L}_{k}^{-}}\right] \forall n \text {; for each } k \in\{1, \ldots, \mathbb{K}\} \text { and } \forall 1 \leq l \leq(p+1) \text {. }
$$

Now, since $\widehat{D}_{k}=O_{p}\left(c_{n_{\bar{K}}^{-}}\right),\left(c_{n_{\mathbb{K}}^{-}} / \widehat{D}_{k}\right) \geq 0$ is stochastically bounded away from 0 . Thus, $\forall k$, and for any given $\varepsilon>0, \exists \delta(k, \varepsilon)>0$ (independent of $n$ ) such that: $\mathbb{P}_{\mathcal{L}_{k}^{-}}\left\{\left(c_{n_{\mathbb{K}}^{-}} / \widehat{D}_{k}\right) \leq \delta(k, \varepsilon)\right\} \leq \varepsilon^{*} \forall n$, where $\varepsilon^{*}=\varepsilon /\{4 \mathbb{K}(p+1)\}>0$. Let $\widetilde{\delta}(\mathbb{K}, \varepsilon)=$ $\min \{\delta(k, \varepsilon): k=1, \ldots, \mathbb{K}\}>0$ (as $\mathbb{K}$ is fixed). Let $\mathbb{A}(k, \varepsilon)$ denote the event: $\left\{\left(c_{n_{\mathbb{K}}} / \widehat{D}_{k}\right) \leq \widetilde{\delta}(\mathbb{K}, \varepsilon)\right\}$, and let $\mathbb{A}^{c}(k, \varepsilon)$ be its complement. Then $\mathbb{P}_{\mathcal{L}_{k}^{-}}\{\mathbb{A}(k, \varepsilon)\} \leq$ $\varepsilon^{*}$, while on $\mathbb{A}^{c}(k, \varepsilon),\left(c_{n_{\mathbb{K}}^{-}} / \widehat{D}_{k}\right)>\widetilde{\delta}(\mathbb{K}, \varepsilon)$. Thus, the bound in (A.10) is dominated by: $2 \exp \left[-M^{2} \widetilde{\delta}^{2}(\mathbb{K}, \varepsilon) /\left\{2(p+1) \mathbb{K} C^{2}\right\}\right]$ on $\mathbb{A}^{c}(k, \varepsilon)$, and trivially by 2 on $\mathbb{A}(k, \varepsilon) \forall k$. Plugging the bound of (A.10) into (A.9) and using all these facts, we then have

$$
\begin{aligned}
& \mathbb{P}\left(\left\|\mathbb{G}_{n, \mathbb{K}}\right\|>M c_{n_{\mathbb{K}}^{-}}\right) \\
& \quad \leq \sum_{k=1}^{\mathbb{K}} \sum_{l=1}^{p+1} \mathbb{E}_{\mathcal{L}_{k}^{-}}\left[2 \exp \left\{-\frac{M^{2} c_{n_{\mathbb{K}}^{2}}^{2}}{2(p+1) \mathbb{K} C^{2} \widehat{D}_{k}^{2}}\right\}\right]
\end{aligned}
$$


(A.11)

$$
\begin{aligned}
& =\sum_{k=1}^{\mathbb{K}} \sum_{l=1}^{p+1} \mathbb{E}_{\mathcal{L}_{k}^{-}}\left[2 \exp \left\{-\frac{M^{2} c_{n_{\mathbb{K}}^{2}}^{2}}{2(p+1) \mathbb{K} C^{2} \widehat{D}_{k}^{2}}\right\}\left\{1_{\mathbb{A}^{c}(k, \varepsilon)}+1_{\mathbb{A}(k, \varepsilon)}\right\}\right] \\
& \leq \sum_{k=1}^{\mathbb{K}} \sum_{l=1}^{p+1}\left[2 \exp \left\{-\frac{M^{2} \widetilde{\delta}^{2}(\mathbb{K}, \varepsilon)}{2(p+1) \mathbb{K} C^{2}}\right\} \mathbb{P}_{\mathcal{L}_{k}^{-}}\left\{\mathbb{A}^{c}(k, \varepsilon)\right\}+2 \mathbb{P}_{\mathcal{L}_{k}^{-}}\{\mathbb{A}(k, \varepsilon)\}\right] \\
& \leq 2 \mathbb{K}(p+1)\left[\exp \left\{-\frac{M^{2} \widetilde{\delta}^{2}(\mathbb{K}, \varepsilon)}{2(p+1) \mathbb{K} C^{2}}\right\}+\varepsilon^{*}\right] \\
& \left.\leq \frac{\varepsilon}{2}+\frac{\varepsilon}{2}=\varepsilon \quad \text { (with some suitable choice } M_{\varepsilon} \text { for } M\right),
\end{aligned}
$$

where the last step follows from noting the definition of $\varepsilon^{*}$ and choosing $M_{\varepsilon}$ to be any $M$ large enough such that $4 \exp \left[-M^{2} \widetilde{\delta}^{2}(\mathbb{K}, \varepsilon) /\left\{2(p+1) \mathbb{K} C^{2}\right\}\right] \leq$ $\varepsilon /\{\mathbb{K}(p+1)\}$. Thus, (A.11) shows $\mathbb{G}_{n, \mathbb{K}}=O_{p}\left(c_{n_{\mathbb{K}}}\right)$ for any fixed $\mathbb{K} \geq 2$. This further establishes (3.11) and all its associated implications. The proof of Theorem 3.2 is now complete.

Acknowledgments. The authors would like to thank Dr. James Robins and Dr. Eric Tchetgen Tchetgen for many helpful discussions throughout the progress of this paper, as well as the editor Dr. Edward George, the anonymous Associate Editor and the two referees for their useful comments and suggestions that helped significantly in improving and revising the original version of this article.

\section{SUPPLEMENTARY MATERIAL}

Supplement to "Efficient and adaptive linear regression in semi-supervised settings" (DOI: 10.1214/17-AOS1594SUPP; .pdf). The supplement includes: (i) Supplementary results for the simulation studies and the real data analysis; (ii) Discussions on generalization of the proposed SS estimators to MAR settings; (iii) Proof of Lemma A.1; (iv) Proof of Theorem 3.1; (v) Proof of Theorem 4.1; and (vi) Proofs of Lemmas A.2-A.3 and Theorem 4.2.

\section{REFERENCES}

ANDREWS, D. W. K. (1995). Nonparametric kernel estimation for semiparametric models. Econometric Theory 11 560-596. MR1349935

Belkin, M., NiYogi, P. and SindhWANi, V. (2006). Manifold regularization: A geometric framework for learning from labeled and unlabeled examples. J. Mach. Learn. Res. 7 2399-2434. MR2274444

Castelli, V. and Cover, T. M. (1995). The exponential value of labeled samples. Pattern Recogn. Lett. 16 105-111.

Castelli, V. and Cover, T. M. (1996). The relative value of labeled and unlabeled samples in pattern recognition with an unknown mixing parameter. IEEE Trans. Inform. Theory 42 21022117. MR1447517 
Chakrabortty, A. and CAI, T. (2018). Supplement to "Efficient and adaptive linear regression in semi-supervised settings." DOI:10.1214/17-AOS1594SUPP.

Chapelle, O., SchÖLKoPf, B. and ZIEN, A. (2006). Semi-Supervised Learning. MIT Press, Cambridge, MA.

Cook, R. D. (1998). Principal Hessian directions revisited. J. Amer. Statist. Assoc. 93 84-100. MR1614584

Cook, R. D. and LeE, H. (1999). Dimension reduction in binary response regression. J. Amer. Statist. Assoc. 94 1187-1200. MR1731482

CoOK, R. D. and WeIsberg, S. (1991). Discussion of "Sliced inverse regression" by K.-C. Li. J. Amer. Statist. Assoc. 86 328-332.

Cozman, F. G. and CoHEN, I. (2001). Unlabeled data can degrade classification performance of generative classifiers. Technical Report No. HPL-2001-234, HP Laboratories, Palo Alto, CA, USA.

Cozman, F. G., Cohen, I. and Cirelo, M. C. (2003). Semi-supervised learning of mixture models. In Proceedings of the Twentieth ICML 99-106.

DuAn, N. and LI, K.-C. (1991). Slicing regression: A link-free regression method. Ann. Statist. 19 505-530. MR1105834

HANSEN, B. E. (2008). Uniform convergence rates for kernel estimation with dependent data. Econometric Theory 24 726-748. MR2409261

KAWAKITA, M. and KANAMORI, T. (2013). Semi-supervised learning with density-ratio estimation. Mach. Learn. 91 189-209. MR3046904

KoHANE, I. S. (2011). Using electronic health records to drive discovery in disease genomics. Nat. Rev. Genet. 12 417-428.

LAFFERTy, J. D. and WASSERMAn, L. (2007). Statistical analysis of semi-supervised regression. Adv. Neural Inf. Process. Syst. 20 801-808.

LI, K.-C. (1991). Sliced inverse regression for dimension reduction. J. Amer. Statist. Assoc. 86 316342. MR1137117

LI, K.-C. (1992). On principal Hessian directions for data visualization and dimension reduction: Another application of Stein's lemma. J. Amer. Statist. Assoc. 87 1025-1039. MR1209564

LIAO, K. P., CAI, T., GAINER, V. et al. (2010). Electronic medical records for discovery research in rheumatoid arthritis. Arthritis Care and Research 62 1120-1127.

MASRY, E. (1996). Multivariate local polynomial regression for time series: Uniform strong consistency and rates. J. Time Series Anal. 17 571-599. MR1424907

NEwEY, W. K. (1994). Kernel estimation of partial means and a general variance estimator. Econometric Theory 10 233-253. MR1293201

NEWEY, W. K., HSIEH, F. and RoBINS, J. (1998). Undersmoothing and bias corrected functional estimation. Technical Report No. 98-17, Dept. of Economics, MIT, USA.

NigAM, K. P. (2001). Using unlabeled data to improve text classification. Ph.D. thesis, Carnegie Mellon University, USA. CMU-CS-01-126. MR2703067

Nigam, K., McCallum, A. K., Thrun, S. and Mitchell, T. (2000). Text classification from labeled and unlabeled documents using EM. Mach. Learn. 39 103-134.

SEEGER, M. (2002). Learning with labeled and unlabeled data. Technical Report No. EPFLREPORT-161327, Univ. Edinburgh, UK.

Sokolovska, N., CAPpÉ, O. and Yvon, F. (2008). The asymptotics of semi-supervised learning in discriminative probabilistic models. In Proceedings of the Twenty Fifth ICML 984-991.

ZhANG, T. and OlES, F. J. (2000). The value of unlabeled data for classification problems. In Proceedings of the Seventeenth ICML 1191-1198.

ZHU, X. (2005). Semi-supervised learning through graphs. Ph.D. thesis, Carnegie Mellon Univ., USA. CMU-LTI-05-192.

ZHU, X. (2008). Semi-supervised learning literature survey. Technical Report No. 1530, Computer Sciences, Univ. Wisconsin-Madison, USA. 
ZHU, L. X. and NG, K. W. (1995). Asymptotics of sliced inverse regression. Statist. Sinica $5727-$ 736. MR1347616

DEPARTMENT OF STATISTICS

UNIVERSITY OF PENNSYLVANIA

3730 WALNUT STREET

JON M. HUNTSMAN HALL, 4TH FLOOR

Philadelphia, PENNSYlVANIA 19104

USA

E-MAIL: achakrabortty@mail.harvard.edu
Department of Biostatistics

HARVARD UNIVERSITY

655 Huntington AVEnue

BUILDING 2, 4TH FLOOR

Boston, Massachusetts 02115

USA

E-MAIL: tcai@hsph.harvard.edu 